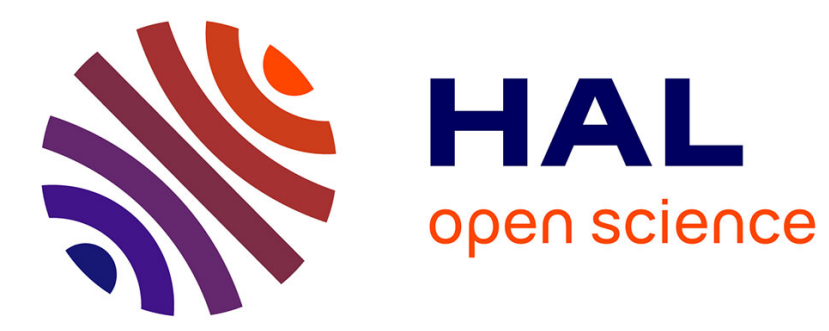

\title{
La perspective pragmatique dans l'étude du raisonnement et de la rationalité
}

Jean-Baptiste van Der Henst

\section{To cite this version:}

Jean-Baptiste van Der Henst. La perspective pragmatique dans l'étude du raisonnement et de la rationalité. Annee Psychologique, 2002, 102, pp.65-108. hal-00000172

\section{HAL Id: hal-00000172 \\ https://hal.science/hal-00000172}

Submitted on 6 Feb 2003

HAL is a multi-disciplinary open access archive for the deposit and dissemination of scientific research documents, whether they are published or not. The documents may come from teaching and research institutions in France or abroad, or from public or private research centers.
L'archive ouverte pluridisciplinaire HAL, est destinée au dépôt et à la diffusion de documents scientifiques de niveau recherche, publiés ou non, émanant des établissements d'enseignement et de recherche français ou étrangers, des laboratoires publics ou privés. 
La perspective pragmatique dans l'étude du raisonnement et de la rationalité. (The pragmatic perspective in the study of reasoning and rationality)

\author{
Jean-Baptiste Van der Henst
}

Université de Louvain (K.U. Leuven, Belgique) / Institut Jean Nicod (Paris)

Adresse :

Jean-Baptiste Van der Henst

K.U. Leuven

Laboratory of Experimental Psychology

102 Tiensestraat, 3000 Leuven, Belgium.

Email : jvanderhenst@hotmail.com ou

Tél. : (00 32) 16326143

Fax : (00 32) 16326099

L'auteur remercie Jean Baratgin, Dick Carter, Yannick Dehée, Benoît Monin, Ira Noveck, Guy Politzer, Sandrine Rossi, Dan Sperber et tous les membres du G.R.I.C.E (Groupe de Recherche sur l'Inférence et la Compréhension Elémentaire) pour leur soutien et leurs commentaires sur des versions précédentes de l'article. L'auteur bénéficie d'une bourse « Marie Curie » de la commission européenne. 


\section{Résumé}

Pour résoudre un problème de raisonnement présenté dans le contexte du laboratoire il faut non seulement représenter les prémisses et en dériver des conclusions mais il faut aussi interpréter les prémisses. Les approches traditionnelles du raisonnement ont rarement pris en compte cet aspect. L'article présente comment la pragmatique linguistique permet de comprendre la manière dont les prémisses utilisées dans les tâches de raisonnement sont interprétées. Il expose plusieurs cadres théoriques et présente une revue détaillée des études qui révèlent l'influence des facteurs pragmatiques dans le raisonnement. En conséquence, l'analyse montre que certaines erreurs observées dans de nombreuses tâches ne sont pas dues à une déficience inférentielle mais résultent d'une divergence entre l'interprétation du sujet et celle de l'expérimentateur.
Abstract.
Solving a reasoning problem not only requires representing a set of premises and deriving conclusions from it but also requires interpreting the premises. This latter point has been rarely taken into account by traditional approaches of human reasoning. The article presents how linguistic pragmatics helps to understand the way premises typically used in reasoning tasks are interpreted. It provides several theoretical frameworks and presents a detailed review of the studies revealing the influence of pragmatic factors in reasoning. As a consequence, the analysis shows that some erroneous answers observed in many tasks are not due to a deficiency in reasoning but result from a discrepancy between the interpretation of the participant and that of the experimenter. 


\section{La perspective pragmatique dans l'étude du raisonnement et de la rationalité.}

\section{Introduction}

Imaginez que l'on vous communique les prémisses suivantes :

Philippe ment plus que Bertrand

Bertrand ment plus que Jean

Si Philippe ment plus que Jean alors tous les électeurs voteront pour Philippe

Certains électeurs sont des pirates.

De ces prémisses vous pouvez déduire que " Philippe ment plus que Jean ", que " tous les électeurs voteront pour Philippe " et que “certains pirates voteront pour Philippe ". Le raisonnement déductif est cette capacité qui permet d'accéder, à partir d'un ensemble d'informations, à d'autres informations qui ne sont pas immédiatement accessibles mais dont la vérité est garantie par la vérité des informations dont elles découlent. Parmi les questions qui intéressent les psychologues, celle de la nature des processus cognitifs à l'œuvre dans le raisonnement est sans nul doute l'une des plus fondamentales. Les psychologues du raisonnement étudient classiquement deux types de processus : les processus de représentation des prémisses et les processus d'inférence. Les processus de représentation sont analogiques selon certaines approches (DeSoto, Handel \& London, 1965 ; Huttenlocher, 1968 ; Johnson-Laird, 1983) ou au contraire, propositionnels selon d'autres (Clark, 1969 ; Anderson \& Bower, 1973). Cette opposition se retrouve au niveau des processus d'inférence. Les partisans de l'approche analogique conçoivent le raisonnement comme une recherche exhaustive de modèles mentaux (Johnson-Laird \& Byrne, 1991) et les partisans de l'approche propositionnelle conçoivent le raisonnement comme l'application de règles d'inférence à la forme propositionnelle des prémisses (Rips, 1994 ; Braine \& O’Brien, 1998).

La question des processus n'est bien sûr pas le seul champ d'investigation de la psychologie du raisonnement. Un autre thème de recherche très prisé est celui des erreurs que commettent les individus lorsqu'ils raisonnent ou, pour être plus précis, lorsqu'ils participent à une tâche de raisonnement. Mises en évidence en particulier dans les travaux de Wason et de Kahneman et Tversky, les erreurs font l'objet de théories spécifiques (Evans, 1989 ; Kahneman, Slovic \& Tversky, 1982) et suscitent un débat philosophique sur la question de la rationalité humaine (Cohen, 1981 ; Stich, 1990 ; Harman, 1995 ; Manktelow \& Over, 1996 ; Evans \& Over, 1997). L'observation d'erreurs dans le raisonnement crée en effet un paradoxe. D'un côté on ne peut que reconnaître la puissance considérable des capacités cognitives et de l'intelligence humaine en général. Mais de l'autre, il est assez facile de 
concevoir des tâches de raisonnement dotées de solutions très simples mais que fort peu d'individus placés dans le contexte d'une expérience parviennent à résoudre. L'observation d'erreurs ou plus exactement d'écarts systématiques par rapport aux modèles normatifs pour des problèmes qui ne dépassent pas la portée de nos capacités cognitives met donc en question la rationalité humaine. Outre les thèmes de recherche qu'ils affectionnent, on peut s'intéresser aux moyens que les psychologues mettent en œuvre pour étudier le raisonnement en laboratoire. Si la psychologie du raisonnement est parfois critiquée pour son manque d'homogénéité théorique (Evans, 1991), elle montre en revanche une parfaite unité sur le plan du matériel et des méthodes utilisés. En effet, le sujet qui participe à une expérience doit toujours raisonner à partir d'énoncés linguistiques (Note 1). Des prémisses sont communiquées par un expérimentateur à un sujet, et en retour celui-ci doit évaluer ou produire une conclusion. Tel est l'immuable scénario d'une tâche de raisonnement présentée en laboratoire. Ce n'est donc pas dans n'importe qu'elle situation que l'on observe le raisonnement, l'élément linguistique est essentiel et le sujet est toujours en situation de communication avec un expérimentateur. Pour que le sujet raisonne sur les informations que l'expérimentateur communique, il lui faut comprendre ces informations et en particulier identifier ce que l'expérimentateur a voulu dire à partir des énoncés linguistiques comme « si p alors q » ou « certains A sont des B ». Aussi, par-delà la question centrale de savoir comment sont représentées les prémisses (i.e. de façon propositionnelle ou analogique), on peut se demander quelles informations les individus représentent à partir de ces prémisses, ou en d'autres termes, comment ils les interprètent.

Curieusement, les psychologues du raisonnement qui se posent cette question sont peu nombreux. Certes, elle ne semble pas toucher directement au raisonnement puisqu'elle est plutôt d'ordre psycholinguistique ou plus précisément d'ordre pragmatique et les processus interprétatifs arrivent en amont des processus proprement liés au raisonnement. Elle est pourtant cruciale car le résultat de l'interprétation constitue ce sur quoi vont porter les processus inférentiels. Si on ne sait pas quelle est l'interprétation des prémisses faites par le sujet, on ne peut déterminer les informations qui servent d'inputs aux processus de raisonnement, on ne peut connaître le cheminement inférentiel que le sujet poursuit et on ne peut juger de la validité de son raisonnement. Le présent article dresse une analyse pragmatico-linguistique du raisonnement fondée sur une revue de la littérature. L'objectif de cette revue est de présenter comment le sujet est susceptible d'interpréter des prémisses qu'il reçoit de l'expérimentateur lors d'une tâche de raisonnement. L' analyse pragmatique permet d'aborder de manière directe le problème de la rationalité. Car la prise en compte de l'étape 
interprétative est une condition nécessaire dans l'attribution d'irrationalité tout autant que peut l'être la prise en compte de l'étape proprement inférentielle.

L'importance de la communication dans le raisonnement.

La pragmatique linguistique étudie les énoncés dans leur situation de communication. Elle analyse donc l'influence du contexte sur l'interprétation des énoncés. Le contexte est constitué de multiples éléments comme les connaissances encyclopédiques, les relations sociales entre les différents interlocuteurs, le moment et le lieu de la situation d'énonciation. Ces éléments vont permettre de donner un sens à l'expression purement linguistique d'une phrase. L'une des premières opérations que le contexte permet est d'identifier les différentes variables ou référents d'un énoncé tels que les pronoms (il, elle...) ou les adverbes de temps et de lieu (ici, bientôt...). La première couche de processus pragmatiques, encore appelés processus pragmatiques "primaires", permet de déterminer une proposition dont il devient possible de dire si elle est vraie ou fausse.

Mais la détermination de cette proposition ne recouvre pas la totalité du message communiqué, qui lui résulte des processus pragmatiques « secondaires ». Précisément, le message communiqué par la proposition dépend du contexte et des conséquences que l'on peut inférer de l'énonciation de cette proposition dans ce contexte. Supposons qu'un petit garçon, Emile, s'entende dire par sa mère «(1) Oh, mais tu n'es vraiment pas un ange aujourd'hui! » et que son père qui vient d'assister à l'échange rajoute « (2) Aujourd'hui, les enfants qui ne sont pas sages seront privés de dessert ». Des énoncés (1) et (2) Emile infèrera qu'il sera probablement privé de dessert. Mais cette inférence n'est possible que si Emile interprète (1) comme « je ne suis pas un enfant sage ». Le seul contenu littéral de (1) (i.e. je ne suis pas un être magique vivant dans le ciel et doté d'une auréole de bonté et de longues ailes blanches...) ne permet pas cette inférence. Pour déclencher une inférence à partir du seul contenu littéral de ces deux prémisses, il faudrait que (1) soit «Oh, Mais tu n'es vraiment pas un enfant sage aujourd'hui » ou alors que (2) soit «aujourd'hui les êtres qui ne sont pas des anges seront privés de dessert ».

De façon générale, l'information communiquée par un énoncé dépasse souvent l'information littérale exprimée par cet énoncé (par exemple l'énoncé 1). Les informations supplémentaires que l'on infère à partir de ce qui est littéralement exprimé dans une certaine situation constituent une grande partie du message communiqué et parfois même la totalité du message communiqué. Bien sûr, ce n'est pas n'importe quelle information qui s'ajoute à ce qui est littéralement exprimé ; si tel était le cas on ne saurait pas ce qui est communiqué. Le 
supplément d'information obéit, comme nous le verrons plus loin, à certaines conventions ou principes qui régissent l'usage de la langue. Quelle en est la conséquence pour l'étude du raisonnement?

Comme déjà signalé, l'étude du raisonnement implique toujours une interaction sociale. Le sujet se trouve dans une relation de communication avec un expérimentateur. Ce dernier se livre à un acte de communication ; il est donc censé respecter les conventions qui régissent l'usage du langage et à ce titre engendre certaines attentes chez le sujet. Précisément, il s'attend à ce que l'expérimentateur observe ces conventions. Mais d'un autre côté, l'expérimentateur incarne la figure du logicien. Ce qui l'intéresse est d'apprécier l'écart existant entre le raisonnement humain et les normes du raisonnement logique. Il pourra donc avoir tendance à utiliser le langage comme un logicien, c'est-à-dire en négligeant en partie les règles de communication, dont la logique n'a que faire. L'interprétation des prémisses chez le sujet risque alors d'être différente de l'interprétation faite par l'expérimentateur. L'erreur que peut commettre l'expérimentateur est de considérer que les processus inférentiels opèrent uniquement sur l'expression littérale des énoncés et non sur l'ensemble de l'information communiquée. Si tel est le cas, la communication entre l'expérimentateur et le sujet risquera d'échouer puisque ce dernier aura fondé son raisonnement sur des informations différentes des informations envisagées par l'expérimentateur. Le raisonnement d'Emile pourrait alors être considéré comme erroné et un « littéraliste » pourrait lui faire valoir que seuls les enfants qui ne sont pas sages seront privés de dessert et que personne n'a dit qu'il n'était pas sage et de conclure : «Emile, tu es irrationnel! ». Cette question de l'attribution d'irrationalité en fonction d'interprétations divergentes se trouve déjà chez Spinoza :

"Lorsque des hommes se trompent dans un calcul, ils ont dans l'esprit d'autres nombres que ceux qu'ils ont sur le papier. C'est pourquoi, si l'on considère leur esprit, ils ne se trompent certes pas ; cependant ils nous paraissent se tromper, parce que nous pensons qu'ils ont dans l'esprit les nombres qui sont sur le papier ; de même, j'ai entendu récemment quelqu'un crier que sa maison s'était envolée sur la poule de son voisin et je n'ai pas cru qu'il se trompait, parce que son intention me paraissait assez claire. Et voilà l'origine de la plupart des controverses : les hommes n'expriment pas correctement leur pensée ou ils interprètent mal la pensée d'autrui. En fait, lorsqu'ils se contredisent le plus, ils pensent les mêmes choses ou bien des choses différentes, de sorte que ce qu'ils considèrent chez 
autrui comme des erreurs et des absurdités n'en est pas" (L'Ethique, p. 402, 2ième partie, Proposition 47).

Chez les psychologues du raisonnement, Mary Henle $(1962 ; 1978)$ est sans doute la première à développer cette question dans l'analyse du raisonnement. Selon elle, l'erreur de raisonnement n'existe pas : "I have never found errors which could be unambiguously attributed to faulty reasoning" (Henle, 1978). La faible performance observée dans certains problèmes vient de ce que le sujet n'arrive pas à se soumettre à l'ensemble des paramètres de la tâche. Pour Henle, les réponses non logiques ne viennent pas d'erreurs dans le raisonnement mais plutôt d'une mauvaise interprétation de la tâche qui conduit à une représentation “ personnelle” de celle-ci. Cette représentation, que l'expérimentateur ne soupçonne pas, diverge de la représentation de la tâche qu'il attribue au sujet. De même, le sujet ne soupçonne pas que la représentation personnelle qu'il se fait de la tâche est différente de la représentation que l'expérimentateur lui attribue. Un véritable problème de communication se pose. Henle (1962), dans une analyse qualitative de certaines tâches, expose les différentes " altérations" que le sujet peut faire subir à une tâche de raisonnement.

La première est une résistance à accepter la tâche. Les sujets s'intéressent moins à l'aspect logique et formel de la tâche qu'à la vérité des prémisses: "They have evaluated the content of the conclusion, not the logical form of the argument" (Henle 1962, page 371, il existe d'ailleurs au sein de la psychologie du raisonnement une littérature importante sur le « biais de croyance » qui montre que les conclusions que nous inférons dépendent en partie du contenu de l'argument ; voir Evans, Newstead \& Byrne, 1993 pour revue). Une autre altération est la " reformulation " d'une prémisse ou d'une conclusion qui conduit à en transformer le sens initial. Deux autres altérations sont l'omission d'une des prémisses ou l'introduction d'une nouvelle prémisse. Henle estime que certains contextes peuvent conduire le sujet à restreindre le matériel expérimental et à ne considérer que les prémisses qui lui paraissent importantes. Il peut à l'inverse introduire une ou plusieurs prémisses qui lui semblent résulter des prémisses précédentes. Dans les deux cas, Henle soutient que le raisonnement doit être évalué en fonction des informations effectivement utilisées par le sujet et non à partir des prémisses fournies par l'expérimentateur.

Henle a donc bien conscience que des phénomènes liés à l'interprétation et à la communication jouent un rôle important dans la résolution d'un problème de raisonnement (Note 2). Néanmoins, elle ne propose pas de théorie précise des phénomènes pragmatiques qui apparaissent lors de la tâche. Elle ne décrit pas de quelle manière les aspects pragmatiques 
du langage contraignent et guident l'interprétation des énoncés. Elle se contente de poser la difficulté dans la communication comme principe explicatif des erreurs. Il est vrai qu'à l'époque, il n'existait pas encore de théorie pragmatique développée que l'on aurait pu appliquer au raisonnement. Il faut attendre les travaux de philosophes et de linguistes tels que Grice, Ducrot, Sperber et Wilson pour qu'une compréhension de l'influence des phénomènes pragmatiques sur le raisonnement commence à voir le jour.

\section{Outils théoriques : les maximes conversationnelles et la théorie de la pertinence.}

2.1. la théorie Gricéenne de la communication.

Concentrons-nous d'abord sur le modèle de Grice (1975), qui est l'approche pragmatique à laquelle il est le plus souvent fait allusion par les psychologues. Selon Grice, tout échange conversationnel entre un locuteur et un destinataire suppose un minimum d'entente, un minimum d'effort coopératif. L'échange entre les deux participants ne suit pas n'importe quelle voie, il implique le respect de règles communes. Grice (1975) soutient que les participants engagés dans un échange sont censés observer un principe de coopération. Ce principe enjoint les participants à ce que leur contribution, au moment de l'échange, soit conforme à la direction et au but exigés par cet échange. Grice précise ce principe par quatre catégories de maximes dites "conversationnelles":

Maximes de quantité

1. Que votre contribution soit aussi informative que nécessaire .

2. Que votre contribution ne soit pas plus informative que nécessaire.

Maximes de qualité

1. Ne dites pas ce que vous croyez être faux.

2. Ne dites pas les choses pour lesquelles vous manquez de preuves.

Maxime de relation

Soyez pertinent.

Maximes de manière

1. Evitez de vous exprimer de façon obscure.

2. Evitez l'ambiguïté.

3. Soyez bref.

4. Soyez ordonné.

Le respect de ces maximes va orienter l'interprétation des énoncés lors de l'échange verbal. Le destinataire, supposant que son interlocuteur est coopératif, devra ajouter des informations au contenu littéral d'un énoncé pour comprendre ce que son interlocuteur communique. Grice 
dénomme ces informations supplémentaires des “implicitations ” (en anglais “implicatures”). Elles se rencontrent classiquement lorsque le locuteur semble enfreindre de façon flagrante une des maximes :

$\mathrm{X}$ : (1) L'article que tu as soumis l'Année Psychologique a-t-il été accepté?

Y: (2) Je n'ai pas écrit la conclusion.

D'après le sens littéral de l'énoncé (2), le locuteur Y semble transgresser la maxime de relation et ne pas se conformer au principe de coopération. Mais le destinataire n'a aucune raison de penser que son interlocuteur, qui est engagé dans un échange conversationnel, ne respecte pas le principe de coopération. Le destinataire peut donc supposer que la transgression de la maxime n'est qu'apparente et que l'énoncé (2) communique (en inférant les implicitations: “ un article sans conclusion n'est pas un article terminé ”, “ un article qui n'est pas terminé ne peut-être soumis à aucune revue ”, “ un article non soumis ne peut être accepté ”) l'information (3): “non, mon article n'a pas été accepté !’ Le sens communiqué, conforme au but exigé par l'échange, est donc différent du sens littéral. Pour faire disparaître la transgression de la maxime, le destinataire doit donc développer un certain nombre d'implicitations qui conduisent à l'énoncé (3). D'autre part, le locuteur doit estimer le destinataire capable de développer ces implicitations. Enfin, le destinataire devra supposer que le locuteur attend de lui qu'il développe ces implicitations. Si la transgression des maximes avait été réelle ou si le locuteur n'avait pas perçu cette transgression comme factice, alors la communication aurait échoué.

La théorie de Grice a eu un impact très important et, comme nous le verrons plus loin, certaines études sur le raisonnement qui s'y réfèrent. Mais cette théorie n'a pas pour vocation la description des phénomènes psychologiques. Sperber et Wilson (1986/1995) montrent d'ailleurs qu'elle est trop intuitive et insuffisamment prédictive sur le plan psychologique (voir aussi Caron, 1983) et que plusieurs aspects méritent d'être éclaircis. Premièrement, à partir d'une proposition énoncée dans un certain contexte, il existe plusieurs interprétations compatibles avec le respect des maximes gricéennes. Pourquoi une seule de ces interprétations a-t-elle été choisie par le destinataire ? Deuxièmement, la procédure de calcul des implicitations décrite par Grice est assez complexe et fait appel à un raisonnement élaboré et conscient qui semble peu compatible avec caractère spontané et automatique de la compréhension. Enfin, la détermination de la pertinence d'un énoncé (maxime de relation) n'est pas spécifiée. Sperber \& Wilson soutiennent que leur notion technique de pertinence et 
les différents principes qui lui sont associés permettent de répondre de façon plus précise à ces questions.

\subsection{La théorie de la pertinence (Sperber \& Wilson 1986/1995)}

Les approches liées à la communication et à la pragmatique sont souvent liées à des questions de philosophie du langage. La théorie de Sperber et Wilson (1986/1995) se distingue des autres approches par le fait qu'elle est aussi une théorie de la cognition et possède donc une dimension psychologique. Au cœur de la théorie, figurent des notions comme celles " d'effort de traitement", “ d'effet cognitif”, de " pertinence ", “d'organisation de la cognition ". On peut, à partir de cette théorie, développer des hypothèses expérimentalement testables. De façon générale, la théorie montre que les processus inférentiels sont guidés par des considérations de pertinence.

La théorie de la pertinence repose sur une idée essentielle qui constitue ce que les auteurs ont appelé le principe cognitif de pertinence ou premier principe de pertinence. Selon ce principe, le but d'un système cognitif comme la cognition humaine, est d'augmenter la connaissance de la manière la plus efficace possible. Pour ce faire, l'individu doit affecter ses ressources au traitement des informations les plus pertinentes de son environnement cognitif. Pour Sperber \& Wilson cela se fait automatiquement, car c'est une propriété fondamentale de la cognition - propriété sélectionnée par l'évolution - que cette tendance à maximiser la pertinence. En d'autres termes, la cognition vise à augmenter l'efficacité cognitive, c'est-à-dire à optimiser ce que l'on pourrait appeler le rapport coûts/bénéfices. Lors d'une activité cognitive comme le raisonnement, le coût peut provenir de la représentation des informations, de la récupération des informations et de la réalisation d'inférences. Les bénéfices qui résultent d'une activité cognitive sont l'acquisition de nouvelles informations, la révision et l'élimination d'informations présentes en mémoire.

Pour définir la pertinence, les auteurs font référence à la notion d'effet contextuel, ou d'effet cognitif. Un effet cognitif est produit par l'interaction d'informations anciennes (C) avec des informations nouvelles $(\mathrm{N})$. Les effets contextuels sont de trois types. Le premier type d'effet contextuel est l'implication contextuelle qui est une information déduite de l'information nouvelle $\mathrm{N}$ dans le contexte $\mathrm{C}$. En ce sens, elle ne pouvait être déduite du seul contexte $\mathrm{C}$ ni de la seule information $\mathrm{N}$. Le second type d'effet contextuel est le renforcement contextuel. Une hypothèse est renforcée lorsque la force dont elle hérite est supérieure à celle qu'elle avait initialement. Le troisième type d'effet contextuel est l'élimination d'hypothèses fausses qui peut résulter d'un conflit entre les informations nouvelles et les informations 
anciennes. Une information sera donc d'autant plus pertinente que ces effets contextuels seront nombreux.

La pertinence d'une information ne dépend pas des seuls effets. Une information fait toujours l'objet d'un traitement qui représente un coût cognitif plus ou moins élevé. L'effort de traitement constitue donc le second facteur qui détermine la pertinence d'une information. Une information sera d'autant plus pertinente que son coût de traitement sera faible. A titre d'exemple, supposons que Jean, souhaite savoir qui des trois sœurs Marie, Julie et Alice a le droit de sortir ce soir. Il sait par ailleurs que seule la plus âgée d'entre elles aura le droit de sortir. Et considérons trois énoncés que l'on pourrait lui communiquer:

(1) Marie est plus âgée que Julie et Alice

(2) Marie est plus âgée que Julie et Alice est plus âgée que Julie

(3) Julie n'est pas aussi âgée que Marie et Alice n'est pas aussi âgée que Marie.

L'énoncé (1) a une certaine pertinence car il permet à Jean de dériver trois effets contextuels : Marie a le droit de sortir, Julie n'a pas le droit de sortir et Alice n'a pas le droit de sortir. L'énoncé (2) est moins pertinent ; il permet juste à Jean d'inférer un seul effet contextuel : Julie n'aura pas le droit de sortir. L'énoncé (3) permet de dériver les mêmes effets contextuels que l'énoncé (1), mais au prix d'un coût de traitement plus élevé. L'énoncé (3) est donc, pour des raisons d'effort, moins pertinent que l'énoncé (1).

Confronté à un stimulus ostensif - qui rend manifeste une intention informative - tel qu'une phrase prononcée par un locuteur lors d'une conversation, le destinataire a une attente de pertinence. En effet, un stimulus ostensif constitue une demande d'attention de la part de celui qui le produit. Le destinataire, n'ayant pas de raison de penser que le locuteur n'est pas coopératif, peut donc supposer que le locuteur a rendu manifeste ce stimulus parce qu'il estime que ce stimulus mérite d'être traité par le destinataire. Pour que le locuteur réussisse à communiquer, il faut que le stimulus produit paraisse pertinent afin que le destinataire y prête attention. Le locuteur doit donc rendre manifeste son intention informative. Il est donc mutuellement manifeste que le locuteur, grâce au stimulus ostensif, rend manifeste au destinataire que le stimulus est pertinent. En d'autres termes, dans tout acte de communication le locuteur communique une présomption de pertinence. Le principe communicatif de pertinence ou second principe de pertinence stipule que tout acte de communication ostensive communique la présomption de sa propre pertinence optimale. La présomption de pertinence optimale (Sperber \& Wilson, 1995, postface, page 271) signifie que le stimulus ostensif produit est suffisamment pertinent pour qu'il mérite d'être traité par le destinataire. Elle signifie d'autre part que ce stimulus est le plus pertinent que le communicateur, en fonction de 
ces capacités et de ses préférences, pouvait produire. Les différents principes de pertinence permettent de caractériser la stratégie de compréhension des énoncés. Selon Sperber \& Wilson, celle-ci consiste à suivre une " route de moindre effort" en considérant les effets cognitifs dans leur ordre d'accessibilité et à “ s'arrêter" lorsque les effets rencontrent les attentes de pertinence.

\section{Les travaux empiriques}

\subsection{Interprétation des prémisses :}

3.1.1. Connecteurs du discours versus connecteurs de la logique.

Bon nombre de problèmes de raisonnement utilisés dans les expériences font intervenir des prémisses qui contiennent des connecteurs (principalement “si...alors ", " et " et " ou ") et des termes quantificateurs (" tous " et "certains "). Une remarque que l'on trouve souvent exprimée, est qu'il n'y a pas de correspondance stricte entre l'usage des connecteurs grammaticaux du langage naturel et celui des connecteurs logiques qui leur correspondent (Caron, 1983, chapitre 14 ; Georges, 1997). D'abord, il existe de multiples connecteurs grammaticaux tels que "comme", “or", "mais", "car", "tandis que” etc., qui n'ont pas de réel correspondant dans le calcul logique des propositions. D'autre part, on associe généralement aux connecteurs logiques $\rightarrow, \wedge, \vee, \leftrightarrow$, les connecteurs linguistiques si...alors, et, ou, si et seulement si, et même pour ces termes, la correspondance est loin de se réaliser. Le contenu véhiculé dans les propositions reliées par un connecteur peut faire varier l'interprétation de celui-ci. Dans la langue, le connecteur semble hériter des relations particulières qui peuvent exister entre les événements décrits par les propositions.

Par exemple, le connecteur de conjonction "et" peut, comme en logique, être symétrique (à savoir que lorsque la proposition " a et b " est vraie la proposition " $b$ et a " est aussi vraie), mais il peut aussi être asymétrique dans son utilisation quotidienne. La propriété d'asymétrie se manifeste lorsqu'un rapport causal ou temporel apparaît entre les deux éléments conjoints. L'énoncé « Jean est mort et il fut enterré » ne communique pas la même chose que l'énoncé « Jean fut enterré et il est mort». Fillenbaum (1971, 1974a), l'un des premiers à s'intéresser à l'usage des connecteurs du langage naturel, a montré que les individus sont sensibles à la différence entre les énoncés où le "et" est asymétrique et les énoncés ou le "et" est symétrique. Il observe un phénomène de normalisation pragmatique. Les sujets confrontés à des phrases où le "et" asymétrique apparaît de manière non naturelle (il fut enterré et il est mort, il s'habilla et il prit sa douche) ont tendance, lors d'une tâche de paraphrase ou de 
reconnaissance à rétablir le sens asymétrique naturel (il est mort et il fut enterré, il prit sa douche et il s'habilla).

De même avec la disjonction, l'usage du terme "ou" dans la langue diffère de l'usage logique de l'opérateur de disjonction “ $\vee$ ”. D'abord, l'emploi du terme "ou" communique souvent une information sur le statut épistémique du locuteur relativement aux deux éléments disjoints. A la différence de la proposition logique $p \vee q$, exprimant qu'il suffit que $p$ soit vraie ou que $q$ soit vraie pour que $p \vee q$ soit vraie, dans le langage naturel l'emploi du terme "ou", dans un énoncé comme $p$ ou q exprime parfois que le locuteur ne sait pas si $p$ ou si $q$ (Fillenbaum, 1977 ; Levinson, 1983) et s'apparente ainsi à un opérateur épistémique (Levinson, 1983). L'emploi du terme “ou” implique d'autre part que les éléments disjoints concernent, dans une certaine mesure, un sujet commun (Fillenbaum, 1974b); en atteste la curiosité d'un énoncé tel que "Paris est pollué ou je mange une glace”. De plus, le "ou" revêt de multiples usages, il peut être inclusif, exclusif et exhaustif, exclusif et non exhaustif. Enfin, comme pour le "et", un énoncé avec “ou” peut être asymétrique notamment dans le contexte des avertissements: “ dépêchez-vous ou vous allez rater le bus ”. Fillenbaum (1974a; b) montre que le "ou" asymétrique est également sujet à la normalisation pragmatique : un énoncé tel que "dépêchez-vous ou vous allez réussir à attraper le bus" est normalisé par la plupart des sujets.

Dans le langage naturel l'utilisation du "Si...alors" diffère également de son correspondant logique $(\rightarrow)$. Un énoncé conditionnel communique parfois des promesses, des avertissements, des menaces ainsi que des relations temporelles ou causales. Fillenbaum (1978) observe que la performance, dans l'évaluation des conclusions d'un syllogisme conditionnel, dépend largement du contenu des propositions $p$ et $q$. L'utilisation d'un énoncé conditionnel dans le langage naturel présuppose qu'il existe un rapport particulier entre l'antécédent et le conséquent; les " $p$ et $q$ " ne sont pas arbitraires à la différence de l'implication matérielle logique qui spécifie la relation conditionnelle entre $p$ et de $q$ uniquement à partir de leur valeur de vérité. La logique définit une table de vérité pour l'implication telle que l'énoncé est faux, si l'antécédent est vrai et le conséquent faux. Pour toutes les autres valeurs de vérité de $p$ et $q$, la proposition $p \rightarrow q$ est vraie. En langage naturel, il est souvent difficile d'accepter comme vrai un énoncé conditionnel dont l'antécédent est faux. La caractérisation vérifonctionnelle de l'implication matérielle ne correspond pas forcément à l'utilisation naturelle d'un énoncé conditionnel. Ainsi, Wason $(1966 ; 1968)$ propose-t-il une table de vérité pour l'implication qui lui paraît la plus plausible : 


\begin{tabular}{ll|l|l}
$\mathrm{p}$ & $\mathrm{q}$ & $\mathrm{p} \rightarrow \mathrm{q}^{*}$ & Si $\mathrm{p}$ alors $\mathrm{q}^{* *}$ \\
\hline Vrai & Vrai & Vrai & Vrai \\
Vrai & Faux & Faux & Faux \\
Faux & Vrai & Vrai & Sans pertinence \\
Faux & Faux & Vrai & Sans pertinence
\end{tabular}

(*table de vérité logique; **table de vérité proposée par Wason)

Une des erreurs souvent observée lors de tâches consacrées à l'étude du raisonnement conditionnel est celle que l'on appelle la négation de l'antécédent. Cette erreur consiste à faire l'inférence suivante:

Si p alors $q$

Or non $p$

Donc non $q$

La logique déductive nous commande de rester indéterminé sur la conclusion et de ne pas conclure "non q". Néanmoins, dans les contextes de menaces et de promesses, des inférences invitées apparaissent (Geis \& Zwicky, 1971; Fillenbaum, 1978) et il devient difficile de ne pas inférer la négation du conséquent à partir de la négation de l'antécédent :

Le locuteur: "Si l'éditeur publie mon article, je lui donne 5000 francs"

Le locuteur: "L'éditeur n'a pas publié mon article”

Le destinataire : "Tu ne lui as donc pas donné 5000 francs".

Cette inférence n'est pas valide, mais il serait injuste de la considérer comme une erreur. Dans la mesure où le locuteur de l'énoncé "si l'éditeur publie mon article je lui donne 5000 francs" s'engage dans un échange conversationnel, il est censé se conformer au principe de coopération. Si le locuteur avait voulu communiquer "même s'il ne publie pas mon article, il existe d'autres raisons qui de toute façon me conduisent à lui donner ces 5000 francs" alors il aurait dû le dire. Dans la mesure où il ne le dit pas, le destinataire doit comprendre que l'énoncé conditionnel véhicule l'implicitation suivante: si l'éditeur publie son article alors le locuteur lui donnera 5000 francs et si l'éditeur ne publie pas l'article alors le locuteur ne lui donnera pas 5000 francs. Le respect du principe de coopération conduit le destinataire à écarter toutes les possibilités autres que la publication de l'article pouvant conduire le locuteur à donner 5000 francs à l'éditeur. Ainsi, le sens littéral de l'énoncé conditionnel sur lequel on 
fonde la validité logique de l'inférence ne correspond pas au sens communiqué auquel doit aboutir le destinataire et qui correspond à l'interprétation d'un énoncé biconditionnel. Or d'après la table de vérité de l'énoncé biconditionnel l'inférence est non pas indéterminée mais bien correcte.

\subsubsection{Implicitations scalaires et informativité}

Les lois d'usage du langage imposent des contraintes d'informativité. On peut exprimer cette exigence d'informativité par la première maxime de quantité de Grice (1975): (“ faites en sorte que votre contribution soit aussi informative que nécessaire (dans le but de l'échange en cours "), par la loi d'exhaustivité de Ducrot (“cette loi exige que le locuteur donne, sur le thème dont il parle, les renseignements les plus forts qu'il possède, et qui sont susceptibles d'intéresser le destinataire ", Ducrot, 1972, page 136) ou encore par le principe de complétude de Begg \& Harris (1982, page 596). Au niveau du raisonnement, on peut retrouver cette exigence. Inférer, à partir d'une prémisse, une conclusion beaucoup moins informative que la prémisse n'a pas un grand intérêt. D'un autre côté, sur le plan logique, rien n'interdit d'inférer à partir d'un énoncé quelque chose de beaucoup moins informatif tant que la validité de l'argument se trouve respectée. Ainsi, une divergence peut-elle exister l'exigence de validité dont rend compte la logique et l'exigence d'informativité dont rend compte la pragmatique. Politzer (1986) développe cette hypothèse et suppose l'existence d'un système cognitif dual dans lequel rentrent en compétition, d'un coté des processus logiques et de l'autre des processus pragmatiques. On peut donc s'attendre, selon cette hypothèse, à ce que les individus rejettent certaines règles logiques qui ne respectent pas la contrainte d'informativité.

Considérons l'exemple de la règle d'introduction de la disjonction:

$$
\text { donc } a \text { ou } b
$$

Dans cette règle, la conclusion véhicule moins d'information que la prémisse. Bien que valide, cette règle d'inférence apparaît étrange et les études expérimentales (Rips, 1983; Braine, Reiser \& Rumain, 1984) montrent que peu d'individus sont enclins à l'accepter. L'étrangeté de cette règle vient bien sûr d'une perte d'information, lorsque l'on passe de la prémisse à la conclusion. L'introduction d'une disjonction dans la conclusion met en doute l'existence de chacun des deux éléments disjoints et notamment l'élément de la prémisse. On passe donc d'une information certaine à une information moins certaine : avec la prémisse, on sait que "a" est le cas, avec la conclusion on met en cause la possibilité que "a" soit le cas en envisageant que "b " soit le cas. Cela revient à inférer " il se peut que a " à partir de " a ". 
On peut également mener une analyse pragmatique de l'utilisation des termes quantificateurs (tous, certains). D'un point de vue logique, il est possible d'inférer à partir d'un énoncé universel, un énoncé particulier. Si le recteur d'une université affirme “ tous les étudiants de l'université ont eu leur examen " il peut a fortiori affirmer " certains étudiants de l'université ont eu leur examen ". Si l'on attribue une propriété à une certaine classe, cette propriété est évidemment valable pour une partie de cette classe. Passer de tous à certains c'est décroître en informativité et donc respecter le cheminement logique puisqu'une conclusion ne peut être plus informative que l'ensemble constitué par les prémisses.

Mais, d'après la première maxime de quantité, un locuteur se doit d'être aussi informatif que possible. Selon cette maxime, si un recteur d'université affirme “ certains étudiants ont eu leur examen ", il peut garantir ce niveau d'informativité ; il n'aurait donc pas exprimé cet énoncé s'il savait que tous les étudiants avaient eu leur examen. L'implicitation qu'il communique est donc que certains étudiants n'ont pas eu leur examen. Il s'agit encore d'un exemple où l'exigence logique diffère de l'exigence communicative. Si l'usage de "certains" peut véhiculer l'implicitation de "certains...ne...pas" et être incompatible avec " tous", le quantificateur logique " certains" est lui compatible avec " tous". En logique, "certains" est la négation de " aucun" et " certains...ne...pas" est la négation de " tous ". Plusieurs travaux expérimentaux (Begg \& Harris, 1982 ; Griggs \& Newstead, 1983 ; Newstead, 1989 ; Politzer, 1990 ; Noveck, 2001) révèlent que l'usage des quantificateurs est soumis à des contraintes pragmatiques. Ces travaux montrent que "certains A sont B" est souvent interprété comme “certains A sont des B, mais pas tous" et que "certains A ne sont pas B " est souvent interprété comme " certains A ne sont pas des B, mais pas aucun".

D’une façon plus générale, l'implicitation “ certains A ne sont pas B ” véhiculé par l'affirmation " certains A sont B " appartient aux implicitations dites "scalaires " (Horn, 1972 ; Levinson, 1983). Une échelle linguisitique (Horn, 1972) comprend un certain nombre de termes qui appartiennent à un même champ sémantique et qui peuvent être ordonnés selon leur informativité : < tous, beaucoup, certains $>$, < réussir, essayer, vouloir $>,<$ devoir, pouvoir $>,<\mathrm{n} \ldots, 3,2,1>$, $<$ certain, probable, possible $>,<$ toujours, souvent, parfois $>$, $<$ et, ou $>$. On peut caractériser l'apparition des implicitations scalaires de la manière suivante: si un locuteur affirme un énoncé qui se situe à un niveau d'une échelle linguistique, il communique implicitement la négation d'un énoncé qui se situe à un niveau supérieur de l'échelle. Car si le locuteur était en position de formuler un énoncé de niveau supérieur, il l'aurait fait (Que votre contribution soit aussi informative que nécessaire, Grice, 1975). Ainsi, 
un locuteur qui affirme "Cadéroussel a 3 cheveux " laisse entendre que Cadéroussel n'a pas 4 cheveux, et il communique donc que Cadéroussel n'a que 3 cheveux. Dans une étude récente Noveck (2001) s'intéresse à l'aspect développemental des implicitations scalaires. Il montre que les enfants ne maitrisent pas bien ces implicitations et adoptent bien souvent une interprétation logique contrairement aux adultes.

L'exigence d'informativité fait que le destinataire attend du locuteur que toute l'information vraie, sue du locuteur, et pertinente pour l'échange en cours, soit exprimée, et que toute l'information fausse soit tue. Dès lors, le destinataire peut inférer que tout ce qui est pertinent pour l'échange en cours et qui n'est pas exprimé est faux. Si un locuteur affirme l'énoncé “certains étudiants ont eu leur examen ” il exprime implicitement que l'énoncé “tous les étudiants ont eu leur examen ” est faux. Il existe donc un rapport entre informativité et vérité qui n'existe pas en logique où une information non exprimée est indéterminée et où une information fausse est automatiquement exprimée par l'opérateur de négation (Politzer, 1986).

\subsubsection{Interprétation et Pertinence : la tâche de sélection}

Lorsque l'expérimentateur présente une tâche de raisonnement, l'expérimentateur oriente (de manière souvent involontaire) la pertinence en manipulant les efforts de traitement et les effets cognitifs. Sperber, Cara et Girotto (1995 ; voir aussi Hardman, 1998 ; Girotto, Kemmelmeier, Sperber \& Van der Henst, 2001) ont appliqué la théorie de la pertinence au paradigme expérimental qui est un des plus étudiés au sein de la psychologie du raisonnement: la Tâche de Sélection. Cette tâche fut conçue par Peter Wason (1966; 1968). Dans cette tâche, le sujet doit déterminer si une règle conditionnelle est vraie ou fausse. Cette règle décrit le contenu de certaines cartes qui comportent une lettre sur une face et un chiffre sur l'autre. La règle est par exemple "S'il y a un A sur une face, il y a un 4 sur l'autre face" (si $p$ alors $q$ ). Pour permettre au sujet d'évaluer la règle, on lui présente quatre cartes dont il ne peut apercevoir qu'une des faces : A, D, 4 et 7 ( $p, q$, non- $p$, non- $q)$. La tâche consiste donc à sélectionner la ou les cartes qu'il est nécessaire de retourner pour déterminer si la règle est vraie ou fausse. Les sujets choisissent en majorité la carte A $(p)$ et les cartes A $(p)$ et $4(q)$ alors que la réponse logiquement correcte correspond à la sélection des cartes $\mathrm{A}(p)$ et 7 (non$q$ ). La carte A doit être choisie car si un nombre autre que 4 apparaît sur l'autre face, alors cette carte contredit la règle. La carte 7 doit aussi être sélectionnée, car si la lettre A apparaît sur l'autre face, alors cette carte réfute également la règle. Par contre, quel que soit le nombre qui apparaît au dos de la carte $\mathrm{D}$, la règle ne peut être réfutée. De même, quelle que soit la 
lettre qui apparaît au dos de la carte 4, la règle ne peut être réfutée (en effet, la règle ne spécifie pas que "s'il y a un 4 il doit y avoir un A ").

L'analyse de Sperber, et al. (1995) montre que les processus mis en jeu dans la résolution de cette tâche ne sont pas des processus que l'on peut assimiler à un raisonnement conscient et démonstratif. L'étude de Sperber, et al. révèle que la sélection des cartes observée dans les multiples études dépend de processus pragmatiques mettant en jeu des inférences non démonstratives inconscientes qui engendrent certaines intuitions de pertinence. Le facteur qui peut modifier la performance n'est pas tant l'utilisation d'un contexte déontique (Griggs \& Cox, 1982 ; Cheng \& Holyoak, 1985 ; pour une application de la théorie de la pertinence au contexte déontique, voir Girotto et al., 2001) ou la formulation négative (Evans, 1982) de la règle conditionnelle, mais les attentes de pertinence provoquées par le contexte de la tâche. La thèse des auteurs est que les sujets réussiront la tâche lorsque le contexte les incitera à vérifier s'il existe des occurrences de $p$ et non $q$. Les sujets échouent lorsque leurs attentes de pertinence ne coïncident pas avec les réponses logiquement correctes.

Selon Sperber et al., la phase d'interprétation de la tâche conduit les sujets à inférer, à partir de la règle conditionnelle et dans un ordre croissant d'accessibilité, les conséquences testables de la règle. Les sujets s'arrêtent lorsque ces conséquences rencontrent leurs attentes de pertinence et sélectionnent donc les cartes qui permettent de tester ces conséquences. Dans l'interprétation d'une règle conditionnelle, trois types d'inférence peuvent être pertinentes. Le premier correspond au déclenchement d'un Modus Ponens. La règle conditionnelle devient pertinente dans la mesure où l'on peut inférer $q$ à partir de $p$. Le second dépend de l'usage pragmatiquement correct d'un énoncé conditionnel. Si l'on emploie un énoncé conditionnel de la forme si p alors $q$, c'est qu'il existe des instances de $p$ et $q$ qui exemplifient la règle. Affirmer “ si $p$ alors $q$ ” alors qu'il n'y a pas de $p$ et $q$ est non pertinent. Or comme tout énoncé communiqué véhicule une présomption de pertinence, on peut inférer qu'il existe des instances de la règle et sélectionner les cartes $p$ et $q$. Le troisième type d'inférence, cette fois plus rare, qui permet de rendre la règle conditionnelle pertinente consiste à interpréter la règle comme exprimant la négation de $p$ et non- $q$. Interpréter la règle de cette manière conduira donc à sélectionner les cartes correctes $p$ et non- $q$. La thèse de Sperber et al. est que la manière dont les sujets interprètent la règle, et la rendent pertinente, peut être orientée très facilement grâce au contexte et au contenu de la règle conditionnelle.

Les auteurs conçoivent une " recette " destinée à élaborer une tâche de sélection où la manière la plus pertinente (le moins d'effort pour le plus d'effets) de comprendre la règle est de l'interpréter comme la négation de $p$ et non-q. Du côté effort, la recette consiste à choisir 
des éléments $p$ et $q$ tels que $p$ et non- $q$ soient plus faciles à représenter que $p$ et $q$. Du côté effet, la recette consiste à imaginer un contexte où $p$ et non- $q$ engendre plus d'effets cognitifs que $p$ et $q$. Cette recette permet théoriquement de créer une infinité de tâches où les sujets réussiront à sélectionner les cartes $p$ et non-q.

Dans une de leurs expériences, Sperber, Cara \& Girotto créent un contexte où le fait d'être un homme $(p)$ et de ne pas être marié (non-q) est plus pertinent que le fait d'être un homme $(p)$ et d'être marié $(q)$. Dans ce contexte, les organisateurs d'une manifestation culturelle ont besoin de volontaires pour garder des enfants. L'un des organisateurs prétend que les hommes célibataires n'ont aucune envie de s'occuper des enfants et il parie que si un volontaire est homme alors il est marié. Dans ce contexte, l'énoncé conditionnel exprime qu'il n'y a pas de volontaire homme $(p)$ et en même temps célibataire (non- $q$ ). La présence de volontaires masculins célibataires est donc plus pertinente que la présence de tout autre volontaire. D'autre part, le concept d'homme non marié est très facile à représenter car dans la langue où l'expérience fut conduite (l'italien), ce concept est lexicalisé par un seul terme ("scapolo"). Les résultats montrent que dans ce contexte $65 \%$ des sujets sélectionnent les cartes $p$ et non-q, alors que dans un contexte où la pertinence se situe au niveau des cartes $p$ et $q$, seulement $16 \%$ des sujets choisissent les cartes $(p)$ et (non- $q)$.

\subsection{Point de vue de l'auditeur et mauvaise compréhension}

Il semble évident que pour réussir à communiquer, un locuteur se doit de prendre en compte le point de vue de son interlocuteur. Pour être compris, il doit considérer ce que le destinataire sait ou ne sait pas, ce qu'il croit ou ce qu'il ne croit pas, ce qu'il est capable ou non de recevoir comme message, etc. Etablir une « base commune » (« common ground» Clark \& Marshall, 1981) est essentiel à la communication. Il existe au sein de la pragmatique expérimentale tout un courant de recherche qui justement vise à étudier la manière dont les individus, lorsqu'ils communiquent, prennent en compte la perspective de leur interlocuteur (on utilise parfois les expressions telles que «perspective taking », voir Krauss \& Fussell 1996 pour une revue, ou « audience design » Clark \& Murphy, 1982). De nombreux travaux empiriques montrent en effet que les individus formulent leur message en fonction des diverses caractéristiques du destinataire, telles que son âge, la langue qu'il parle, son expertise sur le sujet évoqué, ses émotions... A l'inverse, dans le domaine de la psychologie du raisonnement, on observe parfois que les expérimentateurs se comportent bien différemment lorsqu'ils communiquent une tâche, et négligent la perspective de leurs interlocuteurs que sont les sujets. 


\subsubsection{L'inclusion des classes}

Une des grandes questions étudiées par les psychologues du développement, et principalement par Piaget, est celle des inférences faites à partir de l'inclusion des classes. Le problème se pose de la manière suivante: "J'ai cinq marguerites et deux tulipes, est-ce que j'ai plus de fleurs ou plus de marguerites?" Les enfants de moins de huit ans à qui l'on présente cette tâche répondent en majorité qu'il y a plus de marguerites que de fleurs. Ils semblent donc avoir des difficultés à comparer le nombre d'éléments d'une classe (les fleurs) avec celui d'une sous-classe (les marguerites). Selon Piaget, à cet âge, il leur est difficile d'envisager à la fois une partie et le tout qui contient cette partie (Piaget \& Inhelder, 1959). Les enfants se contentent donc de comparer les deux sous-classes entre elles (les marguerites et les tulipes). Cependant, l'approche pragmatique révèle que les résultats obtenus dans les expériences de Piaget ne doivent pas conduire à penser que les enfants ne maîtrisent pas le concept d'inclusion de la partie dans le tout.

On utilise généralement le terme de "fleurs" pour désigner un ensemble composé de plusieurs espèces de fleurs ou pour désigner une espèce spécifique. Le contexte dans lequel on utilise ce terme permet de savoir si l'on fait référence à un ensemble de plusieurs espèces ou à une seule espèce de fleurs. Dans la tâche en question, le terme "fleurs" comporte une ambiguïté référentielle due à l'usage curieux ou pragmatiquement anormal de la question posée. En effet, il est assez étrange de comparer deux niveaux hiérarchiques distincts de la même taxinomie. Habituellement, on fait plutôt des comparaisons entre des classes qui se situent au même niveau hiérarchique (y a-t-il plus de marguerites ou plus de tulipes?) ou des comparaisons entre des classes de niveaux distincts mais qui n'appartiennent pas à la même taxinomie (y a-t-il plus d'animaux ou plus de marguerites?). Le terme "fleurs", tel que l'interprète l'expérimentateur dans la tâche, désigne un ensemble de fleurs comportant les tulipes et les marguerites. Selon cette interprétation, la tâche demande donc une comparaison "verticale" (Politzer, 1993), c'est-à-dire une comparaison de classes à sous-classes, ce qui est inhabituel. Pour faire disparaître la formulation anormale de la question, on peut interpréter "fleurs" comme désignant, non pas la classe des fleurs, mais la sous-classe complémentaire (les tulipes), puisqu'on peut très souvent dénoter une sous-classes par le terme de la classe. Il s'agit d'une comparaison "horizontale" (Politzer, 1993) où deux sous-classes sont comparées.

L'hypothèse de Politzer (1993) est que dans le contexte précis de la tâche, l'interprétation horizontale prévaut chez l'enfant de moins de 8 ans. Mais cela ne signifie pas qu'il s'agit de la seule interprétation que ses capacités cognitives lui permettent de faire. La réponse qu'il formule est en partie déterminée par la représentation de ce que 
l'expérimentateur attend de lui et donc par l'interprétation de la question qu'il lui pose. Le sujet interprète la question de façon à ce que la réponse qu'il formule soit la plus pertinente pour l'expérimentateur. A cet âge, l'enfant veut montrer à l'expérimentateur sa capacité à compter les éléments des différentes sous-classes. Il interprète donc la question comme une demande de comparaison des sous-classes. Après huit ans, la représentation métacognitive de ce que l'expérimentateur attend de lui évolue. Il ne ressent plus le besoin de lui montrer sa capacité à dénombrer et à comparer des sous-classes, estimant certainement que cela est trop facile et ne correspond pas à la demande de l'expérimentateur. A cet âge, la manière la plus pertinente de répondre aux attentes de l'expérimentateur est donc d'effectuer la comparaison verticale.

Pour Politzer, la plupart des modifications expérimentales qui ont produit une amélioration de la performance ont consisté à diminuer l'ambiguïté référentielle; par exemple en remplaçant les noms de classes par des noms de collections (Markman, 1973; Smith \& Rizzo 1982). En effet, alors qu'une classe (les animaux) peut également dénoter une sousclasse (les girafes), une collection (le zoo) ne peut le faire. Il n'y a donc plus d'ambiguïté lorsqu'on utilise des noms des collections. Dans une expérience, menée auprès d'enfants de 5 à 6 ans, Politzer (1993, voir aussi Ahr \& Youniss, 1970) pose une question qui supprime l'ambiguïté référentielle. Dans cette question, le terme de la sous-classe susceptible d'être désigné par le terme de la classe est introduit: "il y a plus de marguerites, plus de tulipes ou plus de fleurs?" De plus, la question est précédée par une tâche de désignation: "montre-moi les marguerites", “montre-moi les tulipes", "montre-moi les fleurs". Les résultats révèlent que le taux de réussite à la question standard est de $15 \%$ et qu'il passe à près de $50 \%$ pour les sujets qui reçoivent la question dont l'ambiguïté a été levée.

\subsubsection{Les tâches de conservation}

La manière dont les enfants, en fonction de leurs capacités métacognitives, conçoivent les attentes de l'expérimentateur, a des implications importantes dans les réponses aux tâches de conservation des nombres (Piaget \& Inhelder, 1959). Dans une des tâches, l'expérimentateur présente aux enfants deux rangées, de même longueur, composées de quatre jetons. Au cours d'une première question, il demande d'abord aux enfants s'il y a plus de jetons dans une rangée que dans l'autre. Puis l'expérimentateur augmente la longueur d'une des rangées en espaçant les quatre jetons. Il demande de nouveau si l'une des deux rangées comporte plus de jetons que l'autre. Il apparaît que les enfants de moins de 7 ans répondent par l'affirmative. On peut alors penser que les enfants sont incapables de conserver les 
nombres et qu'une augmentation de la longueur correspond pour eux à une augmentation du nombre de jetons.

Certains travaux (McGarrigle \& Donaldson, 1975; Donaldson, 1982) montrent cependant que les sujets considèrent l'augmentation de la longueur d'une des deux rangées comme un élément déterminant pour résoudre la tâche. Ils accordent donc une importance à une action produite par l'expérimentateur qui en fait n'en a aucune. Cela ne signifie pas pour autant qu'ils estiment le nombre de jetons plus grand dans l'une des rangées. D'après McGarrigle \& Donaldson (1975), les deux questions pourtant identiques, n'ont pas été interprétées de la même manière. En accordant une importance à la modification introduite par l'expérimentateur, les sujets réinterprètent la seconde question comme signifiant "y a-t-il une rangée plus longue que l'autre?” Dans leur étude, McGarrigle \& Donaldson (1975) modifient la structure intentionnelle de la tâche. Ils font croire aux sujets que l'augmentation de la longueur n'est plus le fait de l'expérimentateur mais qu'elle est purement accidentelle. Dans cette nouvelle condition expérimentale, les auteurs observent une augmentation très nette de la performance. Les sujets ont donc tendance à ne plus considérer l'augmentation de la longueur comme un élément qu'il faut prendre en compte pour résoudre la tâche, pour la simple raison qu'elle est indépendante des intentions de l'expérimentateur.

\subsubsection{L'effet de suppression}

Byrne, dans une étude souvent citée (Byrne, 1989), prétend avoir montré que l'une des inférences valides les plus communément effectuées, à savoir le Modus Ponens (Si $p$ alors $q$; or $p$; donc $q$ ), pouvait facilement être désamorcée en transformant le contexte. La manipulation expérimentale de Byrne consiste à présenter, avant la prémisse catégorique, non pas une, mais deux prémisses conditionnelles:

(1) Si elle a une dissertation à écrire alors elle travaillera tard à la bibliothèque.

(2) Si la bibliothèque reste ouverte alors elle travaillera tard à la bibliothèque.

(3) Elle a une dissertation à écrire.

Les résultats montrent en effet que seuls 38\% des sujets effectuent un Modus Ponens à partir du premier énoncé conditionnel et de la prémisse catégorielle pour dériver la conclusion:

(4) donc elle travaillera tard à la bibliothèque.

L'hypothèse de Byrne est que si les sujets n'ont pas effectué cette inférence, c'est que la règle de Modus Ponens n'est pas présente chez l'être humain, en tant que règle d'inférence mentale (Braine \& O’Brien, 1998 ; Rips, 1994). 
Pour accepter le point de vue de Byrne, il faut savoir quelles informations les sujets soumettent à la règle de Modus Ponens. Si en effet, ils cherchent à appliquer la règle du Modus Ponens uniquement à la première prémisse conditionnelle et à la prémisse catégorique, alors le résultat de Byrne met en défaut les capacités inférentielles des sujets et met en doute le fait qu'il existe une règle d'inférence mentale correspondant au Modus Ponens.

L'analyse pragmatique menée par Politzer \& Braine (1991, voir aussi Fillenbaum, 1993) va à l'encontre de cette position. L'énoncé (1) présuppose bien entendu que la bibliothèque sera ouverte. En effet si le locuteur, censé être coopératif, savait que la bibliothèque était susceptible d'être fermée alors il n'aurait pas exprimé (1). Par contre, l'énoncé (2) contredit ce que présuppose l'énoncé (1). Si le locuteur, censé être coopératif, n'avait pas des doutes sur la possibilité que la bibliothèque soit fermée, alors il n'aurait pas affirmé (2). L'énoncé (2) remet donc en cause (1), et laisse entendre que Marie peut avoir une dissertation à écrire sans rester tard à la bibliothèque. De plus, la condition que la bibliothèque soit ouverte apparaît comme une condition "plus nécessaire" (Chan \& Chua, 1994) que la condition d'avoir une dissertation à écrire pour que Marie travaille tard à la bibliothèque. Dès lors qu'il y a un doute sur l'ouverture de la bibliothèque il devient impossible de produire la conclusion (4). Les prémisses (1) et (2) communiquent donc (Braine \& O'Brien, 1991) :

(5) Si elle a une dissertation à écrire et si la bibliothèque est ouverte, alors elle travaillera tard à la bibliothèque.

L'application de la règle du Modus Ponens aux prémisses (3) et (5) interdit bien entendu la conclusion (4).

\subsubsection{L'omission des taux de base}

Amos Tversky et Daniel Kahneman ont conçu un grand nombre de problèmes qui testent l'utilisation des « taux de base » dans le jugement probabiliste (voir Koehler, 1996 pour une revue). Voici un de ces problèmes :

Considérez les données suivantes à propos du suicide. Dans une population de jeunes adultes, $80 \%$ des individus sont mariés et $20 \%$ sont célibataires. Le pourcentage de morts par suicide est trois fois plus élevé chez les personnes célibataires que chez les personnes qui sont mariées.

Quelle est la probabilité pour qu'un individu, choisi au hasard parmi ceux qui se sont suicidés, soit célibataire? 
Les résultats obtenus par Tversky \& Kahneman (1980) montrent que la majorité des sujets évaluent la probabilité que l'individu soit célibataire à 0.75 . Ces sujets semblent négliger le fait que seulement $20 \%$ de la population est célibataire (information sur les taux de base) et ne fonder leur réponse que sur l'information diagnostique (troisième phrase du problème).

L'hypothèse de Macchi (1995) à est que l'omission des taux de base peut provenir d'une mauvaise compréhension des données du problème. Pour elle, la manière dont le problème est présenté peut inciter le sujet à croire que les informations sur les taux de base et l'information diagnostique ne sont pas indépendantes. Selon Macchi, l'information diagnostique, telle qu'elle est communiquée, peut laisser croire qu'elle intègre les taux de base. Le sujet peut donc considérer qu'il n'a plu besoin de les prendre en compte. Ainsi, l'information diagnostique (1) “le pourcentage de morts par suicide est trois fois plus élevé chez les personnes célibataires que chez les personnes qui sont mariées ”, pourrait être interprétée comme signifiant (2) “parmi les gens qui se sont suicidés, le pourcentage de personnes célibataires est trois fois plus élevé que celui de personnes mariées ". Selon Macchi, il existe deux manières d'inciter les individus à considérer l'information diagnostique comme indépendante des taux de base. La première consiste à modifier la question posée, la seconde consiste à modifier l'information diagnostique.

Dans sa première expérience, Macchi remplace la question initiale par la question suivante :

Quelle est la probabilité pour qu'une personne qui s'est suicidée, choisie au hasard parmi la population des jeunes adultes, soit célibataire?

Dans la version initiale, la question ne réfère pas à la population générale (celle des jeunes adultes) mais à une sous-classe (celle des personnes qui se sont suicidées). D'après Macchi, la référence à la sous-classe seule signifie que l'information diagnostique intègre les taux de base. Dans la nouvelle version, la référence à la classe est explicite, ce qui a pour conséquence de bloquer l'interprétation (2). Les résultats montrent que dans version initiale du problème, seuls $34 \%$ tiennent compte des taux de base, alors qu'ils sont $62 \%$ dans la nouvelle version.

Dans la troisième expérience la modification touche cette fois directement à l'information diagnostique qui est énoncée de la manière suivante :

Le pourcentage de morts par suicide est de 1\% chez les personnes célibataires et de 3\% chez les personnes qui sont mariées. 
Macchi estime que cette modification empêche de considérer l'information diagnostique comme référant seulement à l'ensemble des personnes qui se sont suicidées. Dans cette version, les individus qui se suicident, sont présentés comme une partie de la population, à savoir les gens qui sont mariés et les célibataires. Les résultats montrent que dans cette condition $90 \%$ des sujets prennent en compte des taux de base dans leur jugement.

\subsection{Des problèmes conçus pour tromper.}

On peut critiquer les études présentées dans la section précédente dans la mesure où l'expérimentateur néglige la perspective et l'interprétation du sujet. Dans les études qui suivent, il franchit un pas de plus. L'expérimentateur semble avoir parfaitement conscience du point de vue du sujet mais l'utilise pour mieux l'induire en erreur. La recette consiste à faire croire que des informations sont pertinentes pour résoudre le problème alors qu'elles ne le sont pas. Tout ce que ces études risquent de montrer, c'est que les expérimentateurs sont en effet capables de tromper leurs sujets en violant le principe communicatif de pertinence (Sperber \& Wilson, 1995). Le problème qui se pose est évidemment que le sujet n'a aucune raison de penser qu'il va être induit en erreur par un scientifique qui est censé étudier ses capacités inférentielles mais non ses capacités à traiter des informations communiquées en faisant fi de leur présomption de pertinence.

\subsubsection{Les ingénieurs et les avocats}

Un paradigme très connu dans le domaine du jugement probabiliste est le problème des “ ingénieurs et des avocats " (Kahneman \& Tversky, 1973) qui lui aussi teste l'utilisation des taux de base. On présente aux sujets le portrait suivant:

“Jean est un homme de 45 ans. Il est marié et a quatre enfants. Il est en général conservateur, prudent et ambitieux. Il ne s'intéresse pas aux questions politiques et sociales et consacre la plupart de son temps libre à ses nombreux passe-temps tels que la menuiserie, la voile et les énigmes mathématiques".

Dans une condition, on informe certains sujets que le portrait a été tiré au hasard parmi un ensemble de 30 ingénieurs et 70 avocats et, dans une autre condition, on informe les sujets que le portrait a été tiré au hasard parmi un ensemble de 70 ingénieurs et 30 avocats. Les résultats montrent que dans les deux conditions les sujets estiment avec la même probabilité 
que le portrait est celui d'un ingénieur. Ils ne prennent donc pas en compte les informations sur les taux de base. Selon Kahneman et Tversky, ce résultat montre parmi d'autres, que les sujets n'obéissent pas aux principes probabilistes mais qu'ils suivent des stratégies heuristiques, efficaces dans certaines situations et inefficaces dans d'autres. Dans le cas présent, il s'agit de l'heuristique de réprésentativité. Elle consiste à évaluer le degré de similarité entre deux événements. Dans le problème des ingénieurs et des avocats les sujets fondent leur jugement sur le degré similarité entre la représentation qu'ils ont de l'ingénieur type et le portrait présenté.

Depuis cette première étude, beaucoup d'autres travaux (voir Koelher, 1996 et Baratgin, 1999, pour revues) révèlent cependant que, dans certaines circonstances, les sujets sont capables d'utiliser les taux de base. Hilton (1995, voir aussi Baratgin, 1999), faisant référence à plusieurs études, montre notamment que des aspects pragmatiques jouent un rôle important dans l'utilisation ou non des taux de base. Par exemple, lorsque les taux de base sont présentés avant le portrait, ayant ainsi un poids informatif plus élevé que s'ils sont présentés après, les sujets les prennent plus facilement en compte (Krosnick, Li \& Lehman, 1990).

Dans une autre étude, Schwartz, Strack, Hilton \& Naderer (1991) font varier la structure conversationnelle de la tâche en en modifiant la perspective intentionnelle. Dans une condition (similaire à l'étude de Kahneman \& Tversky, 1973), l'expérimentateur informait les sujets que le portrait avait été constitué par des psychologues et que d'autres psychologues avaient dû effectuer la même tâche qu'eux. D'après les auteurs, ce problème apparaît plus comme un problème de psychologie que comme un problème de statistiques. Cette condition souligne l'importance des informations psychologiques fournies dans le portrait au détriment des informations sur les taux de base. Elle conduit le sujet à penser que l'expérimentateur, en vertu des règles de communication, attend de lui qu'il se fonde sur ces informations pour effectuer la tâche. Il suffirait donc d'atténuer l'importance des informations véhiculées dans le portrait pour que les taux de base soient plus facilement pris en compte. Ainsi, dans une autre condition, les sujets apprenaient que le portrait avait été constitué non plus par des psychologues mais par des chercheurs et que, point important, des statisticiens avaient dû effectuer la même tâche qu'eux. Cette condition, sans changer le contenu du portrait, donne un poids plus important à l'information sur les taux de base et tend à transformer le problème en un problème de statistique. Elle peut conduire le sujet à penser que l'expérimentateur attend de lui qu'il utilise les taux de base. Enfin, dans une autre condition, les sujets apprenaient que les informations psychologiques qui constituent le portrait avaient été réunies au hasard par un ordinateur à partir des fichiers détenus par un psychologue. Dans cette 
condition les informations qui constituent le portrait ont été produites non pas de façon intentionnelle par un être humain, mais de façon aléatoire par un ordinateur. Les règles standard de la communication humaine s'appliquent donc de manière moins stricte. Dans les trois conditions, les informations présentées dans le portrait correspondaient à celui d'un ingénieur alors que la proportion d'ingénieurs était de 30 pour 100. Les résultats montrent que les sujets négligent beaucoup plus l'information sur les taux de base dans la condition "psychologues" que dans les conditions "statisticiens" et "ordinateurs".

\subsubsection{L'erreur de conjonction}

La théorie des probabilités stipule que la probabilité d'un événement ne peut être plus petite que la probabilité de cet événement avec la conjonction d'un autre événement: $\mathrm{P}(\mathrm{A}) \geq \mathrm{P}$ (A \& B). Une étude très connue de Tversky \& Kahneman (1983) montre que les sujets semblent violer ce principe. On présente aux sujets le portrait de Linda:

Linda a 31 ans, elle est célibataire, elle ne mâche pas ses mots et c'est une personne très brillante. Elle a un diplôme de philosophie. Etudiante, elle se sentait très concernée par les problèmes de discrimination et de justice sociale et elle a également participé à des manifestations antinucléaires.

On demande ensuite aux sujets de décider laquelle des propositions suivantes est la plus probable:

(1) Linda est employée de banque.

(2) Linda est employée de banque et milite dans un mouvement féministe. $89 \%$ des sujets estiment la probabilité de (2) supérieure à la probabilité de (1). Pour Tversky $\&$ Kahneman (1983), les sujets suivent encore une fois l'heuristique de représentativité. Le portrait actuel de Linda, employée de banque et militante féministe, est le plus représentatif du personnage qu'était Linda dans sa jeunesse.

Plusieurs chercheurs (Dulany \& Hilton 1991; Politzer \& Noveck; 1991) ont mené une analyse pragmatique de la tâche et montrent que la comparaison de $A \& B$ avec $A$ communique en fait, par un calcul d'implicitation, la comparaison de $A \& B$ avec $A \&$ non- $B$. Pour faire disparaître l'étrangeté qui consiste à comparer une classe A (Linda est employé de banque) à une de ces sous-classes A \& B (Linda est employé de banque et milite dans un mouvement féministe), les sujets interprètent en fait A comme la sous-classe (A \& non-B) et comparent donc, non pas une classe avec une sous-classe, mais deux sous-classes: A \& B 
avec A \& non-B. Dulany \& Hilton (1991) estiment que les sujets considèrent que l'expérimentateur détient un savoir important sur le personnage de Linda. Ils peuvent ainsi supposer que si l'expérimentateur ne dit pas dans l'énoncé (1) que Linda est une militante féministe, c'est que l'expérimentateur sait que tel n'est pas le cas, il communique donc l'implicitation que Linda n'est pas une militante féministe. Si les sujets font cette interprétation, alors juger (2) comme étant plus probable que (1) n'est plus en contradiction avec le principe probabiliste. Dans leurs expériences, Dulany \& Hilton (1991) demandent aux sujets quelle interprétation ils ont de l'énoncé (1). Les sujets qui développent une interprétation dépendante des contraintes pragmatiques ne peuvent être considérés comme commettant l'erreur de conjonction. Il apparaît que parmi les sujets qui ont une interprétation de (1) libre des contraintes pragmatiques (c'est-à-dire qui n'interprètent pas l'énoncé 1 comme signifiant A \& non B), seuls 23\% font l'erreur de conjonction. Les auteurs en concluent que c'est à ces seuls ces sujets que l'on peut attribuer l'erreur de conjonction.

\subsubsection{Le problème $2-4-6$}

Les processus d'induction et les processus de test d'hypothèse ont souvent été étudiés dans le cadre d'un autre paradigme, lui aussi très célèbre, de Peter Wason : le problème 2-4-6 (Wason, 1960). L'expérimentateur a en tête une règle qui permet d'engendrer des séquences de trois nombres. Un exemple de triplet engendré par cette règle est la séquence 2-4-6. La tâche consiste à découvrir la règle précise que l'expérimentateur a en tête en proposant différents triplets. Pour chaque triplet propose, l'expérimentateur indique s'il est ou non conforme à la règle. Lorsque le sujet pense avoir trouvé la règle, il en informe l'expérimentateur. Si la règle qu'il annonce est différente de celle de l'expérimentateur, alors le sujet doit recommencer à proposer des séquences de nombres jusqu'à ce qu'il annonce une nouvelle règle, et ainsi de suite. La règle à découvrir est "trois nombres croissants". L'écart existant entre les trois nombres n'a donc aucune importance. La découverte de la règle pose beaucoup de difficultés. Dans l'expérience de Wason (1960), six sujets seulement sur 29 ont réussi à trouver la règle du premier coup. Et certains d'entre eux n'arrivent jamais à trouver la règle. Les sujets ont en tête des règles assez spécifiques dont ils ont beaucoup de mal à s'affranchir : trois nombres pairs croissants consécutifs; trois nombres s'accroissant avec un intervalle de 2 ; trois nombres s'accroissant avec un intervalle identique, etc. Ces règles engendrent des triplets qui sont aussi en conformité avec la règle très générale de l'expérimentateur. Les sujets reçoivent donc toujours un retour d'information positif lorsqu'ils proposent leurs triplets. La stratégie des sujets est de proposer des exemples positifs 
confirmant la règle qu'ils ont eux en tête sans proposer d'exemples négatifs pouvant la réfuter. Or pour réussir la tâche, il faut nécessairement proposer des exemples négatifs (ou “éliminatifs" selon la terminologie de Wason, 1960 ). Les résultats montrent en outre que certains sujets, qui ont annoncé une règle qui a été contredite par l'expérimentateur, proposent parfois à plusieurs reprises, la même règle mais formulée différemment.

Wason interprète ces résultats comme la manifestation d'un biais de confirmation. Les sujets s'efforcent de trouver des exemples qui confirment la règle qu'ils ont eux en tête, sans essayer de la réfuter. Evans (1989) pense qu'il s'agit d'un “ biais de positivité ”. Pour Evans, l'explication n'est pas que les sujets cherchent à confirmer leur règle, mais qu'ils sont tout simplement incapables de tester leur règle avec des exemples négatifs. Klayman \& Ha (1987) estiment que les sujets développent une stratégie de test positif. Cette stratégie consiste simplement à considérer plutôt les cas qui ont la propriété attendue (par exemple “ être un triplet de nombres pairs croissants ") que les cas qui ne l'ont pas.

L'approche pragmatique (Politzer, 1991) ne permet pas d'expliquer pourquoi les sujets testent toujours positivement leur hypothèse, mais elle permet de comprendre pourquoi ils testent tel type d'hypothèse et pourquoi ils ont des difficultés à s'affranchir de leur hypothèse. Le triplet 2-4-6 se situe à un certain niveau de spécificité, non représentatif de la généralité de la règle de l'expérimentateur (trois nombre croissants). Mais le sujet peut considérer que l'expérimentateur attend de lui qu'il découvre une règle qui hérite en partie de la spécificité de l'exemple. Si l'expérimentateur, qui s'engage dans un échange conversationnel et qui donc se présente comme coopératif, avait voulu éviter de communiquer une règle générale, il l'aurait fait en proposant un exemple plus anodin (5-8-23). Comme il ne le fait pas, c'est que cet exemple a sa raison d'être et que la spécificité qu'il véhicule est nécessaire pour résoudre la tâche. Le sujet est en droit de penser que le triplet 2-4-6 est le plus pertinent que l'expérimentateur, en fonction de ses capacités, pouvait lui communiquer pour découvrir la règle. Mais l'expérimentateur, lui, ne respecte pas le principe communicatif de pertinence (Sperber \& Wilson, 1995) selon lequel un énoncé communique la présomption de sa propre pertinence optimale. Le sujet est en droit d'utiliser toute la spécificité de l'information pour résoudre le problème, il serait même pragmatiquement anormal de ne pas le faire et de commencer par tester la règle " trois nombres croissants". La difficulté de la tâche ne réside donc pas dans la complexité de la règle mais dans l'aspect non coopératif de l'échange conversationnel.

On pourrait augmenter la performance en permettant au sujet de constater que l'exemple 2-4-6 communique un niveau de spécificité plus élevé que celui de la règle que 
l'expérimentateur a en tête. Dans la quatrième expérience de l'étude de Tweney, Doherty, Worner, Pliske, Mynatt, Gross \& Arkkelin (1980), que Peter Wason a suggéré aux auteurs, l'expérimentateur a, non pas une, mais deux règles en tête : une règle pour les triplets de type “DAX" (trois nombres croissants) et une règle pour les triplets de type "MED" (tous les autres triplets). Les sujets doivent découvrir ces deux règles. Le triplet 2-4-6 leur est donné comme un exemple de DAX. Par rapport au paradigme initial de Wason (1960), lorsque les sujets proposent un triplet, Tweney et al. remplacent donc la réponse " oui ce triplet confirme la règle " par la réponse “c'est un DAX" et la réponse " non ce triplet ne confirme pas la règle ” par la réponse “ c'est un MED ”. Supposons que le sujet fasse l'hypothèse que la règle pour les DAX soit “ trois nombres pairs croissants avec un intervalle de 2 ”. Le sujet peut faire un test positif de la règle pour les DAX et proposer 6-8-10 ; l'expérimentateur lui répondra “DAX". Et supposons qu'il fasse ensuite l'hypothèse que la règle pour les " MED " est “ trois nombres impairs croissants avec un intervalle de 2 ”. Le sujet peut faire un test positif de la règle pour les MED et proposer 1-3-5 ; l'expérimentateur lui répondra non pas “MED " mais “DAX”. Une tentative de test positif de la règle concernant les MED correspond en fait à un test positif de la règle concernant les DAX. Le sujet, en croyant tester un MED, teste un DAX. Il devra donc réviser son hypothèse sur les DAX et envisager une nouvelle hypothèse plus générale, compatible avec les deux triplets. Le sujet peut alors se rendre compte que la spécificité de l'exemple 2-4-6 n'est pas à la hauteur de la spécificité de la règle concernant les DAX, et que l'expérimentateur l'a en quelque sorte induit en erreur. Le sujet sera donc enclin à moins se focaliser sur les traits spécifiques du triplet 2-4-6 (voir Rossi, Caverni \& Girotto, 2001 pour une analyse plus récente). Les résultats obtenus dans l'étude de Tweney et al. montrent un accroissement très net de la performance. Soixante pour cents des sujets ont réussi à trouver les deux règles du premier coup, alors que dans l'étude de Wason, à peine plus de $20 \%$ des sujets ont réussi à découvrir la règle du premier coup.

Bien sûr il existe certainement d'autres travaux empiriques à effectuer afin de tester plus directement l'hypothèse pragmatique. On peut notamment envisager des situations où la structure conversationnelle de la tâche incite les sujets à considérer que la découverte d'une règle non spécifique est plus pertinente que la découverte d'une règle spécifique malgré la présence d'un exemple spécifique. 


\section{Intégration de la pragmatique dans les théories classiques du raisonnement}

Les défenseurs des deux principales théories du raisonnement, la théorie des modèles mentaux (Johnson-Laird, 1983; Johnson-Laird \& Byrne, 1991) et la théorie des règles d'inférence mentale (Braine, 1990; Braine \& O'Brien, 1998; Rips, 1994), ont laissé une place assez limitée à la pragmatique même si les défenseurs de la théorie des règles reconnaissent son importance. Ces deux théories sont opposées l'une à l'autre, et de nombreuses études conceptuelles et expérimentales issues des deux camps alimentent le débat qui les oppose (Rips, 1986; Johnson-Laird, Byrne \& Schaecken, 1992; Bonatti, 1994a ; b; O'Brien, Braine \& Yang, 1994; Johnson-Laird, Byrne \& Schaecken, 1994; Girotto, 1995 ; Oléron, 1995 ; Noveck \& Politzer, 1998).

D'après l'hypothèse de la logique mentale, l'esprit humain dispose d'un ensemble de règles d'inférence qu'il applique à la forme propositionnelle des informations traitées. A un niveau assez théorique (Bonatti, 1994b ; Braine, 1990 ; Braine \& O’Brien, 1991) les défenseurs de la logique mentale reconnaissent l'importance et la nécessité de l'analyse pragmatique. En particulier, ils font parfois appel à la pragmatique, pour expliquer l'apparition des erreurs et des effets liés au contenu (Braine \& Rumain, 1983 ; Rumain, Connell \& Braine, 1983). Plusieurs chercheurs affirment que la logique mentale ne permet d'expliquer ni les erreurs ni les effets de contenu. En effet, comme les règles d'inférence sont toutes supposées valides, les erreurs de raisonnement ne devraient pas exister. D'autre part, comme l'application de règles d'inférence est un processus syntaxique, et donc indépendant du contenu exprimé par les prémisses, la performance pour des problèmes formellement équivalents, même avec des contenus différents, devraient être identiques. Or il n'en est rien, des études montrent que le contenu des prémisses modifie la performance dans bon nombre de tâches (par exemple dans la tâche de sélection de Wason). Selon les défenseurs des approches à base de règles, l'application des règles ne se fait pas sur la forme propositionnelle des prémisses, mais sur les représentations - aussi de nature propositionnelle - qui sont communiquées par les prémisses (Noveck, Lea, Davidson, \& O’Brien, 1991). Les effets de contenu apparaissent car un certain contenu va communiquer plus d'information qu'un autre contenu et va donc convoquer des règles d'inférence différentes. De même, les erreurs se manifestent lorsque l'application des règles s'effectuent sur des informations communiquées qui aboutissent à des conclusions qui diffèrent des conclusions auxquelles conduit l'application de règles à la stricte représentation sémantique. Les défenseurs des approches à base de règle s'efforcent-ils donc de contrôler les facteurs pragmatiques et de concevoir des expériences où les prémisses ne vont pas entraîner d'implicitation conversationnelle (Braine, 
Reiser \& Rumain, 1984 voir cependant O'Brien \& Davidson, 1989 qui usent d'un langage ouvertement logique) permettant ainsi de faire coïncider l'interprétation de l'expérimentateur et celle du sujet.

Si certains des partisans de la logique mentale se réfèrent à un niveau théorique à la pragmatique et en particulier au modèle de Grice, ils ne proposent cependant pas d'études expérimentales précises intégrant à la fois le processus d'interprétation et le processus d'application de règles. D'autre part, des questions sur le choix d'application de certaines règles et sur la direction du cheminement inférentiel restent en suspens. Dans la vie quotidienne, à la différence des expériences de laboratoire, l'activité inférentielle des individus les conduit rarement à évaluer des conclusions en fonction de certaines prémisses ou à produire n'importe quelle conclusion à partir d'un ensemble de prémisses donné. Ils cherchent en général à accroître leur connaissance à partir d'informations à leur disposition et ce dans un but précis. Ils ne vont donc pas essayer d'obtenir tout ce qui peut être inféré à partir d'un certain ensemble d'informations. En fonction de leur objectif du moment, ils vont suivre des cheminements inférentiels plus ou moins divergents. Ainsi, par rapport à un ensemble d'informations, les attentes de pertinence pourront être différentes. Il peut être utile d'étudier comment un système de règles va opérer avec des contraintes pragmatiques. Le principe cognitif de pertinence offre une réponse possible. On peut penser que le système de règles opère de manière à maximiser la pertinence des informations auxquelles il s'applique.

La théorie des modèles mentaux développée par Johnson-Laird (Johnson-Laird, 1983 ; Johnson-Laird \& Byrne, 1991) est l'approche directement concurrente de la théorie des règles. Selon cette approche, les individus construisent un modèle mental des prémisses qui n'est autre qu'une représentation analogique de la situation décrite dans les prémisses. A partir de ce modèle, ils produisent une information qui n'est pas explicitement exprimée dans les prémisses. Puis, ils cherchent à évaluer cette conclusion en construisant d'autres modèles (ou “ modèles alternatifs") des prémisses susceptibles de réfuter la conclusion initiale. Il mettent ainsi en œuvre une procédure de réfutation (falsification procedure).

Deux aspects de la théorie des modèles mentaux peuvent être complétés par une approche pragmatique. Une première façon de compléter la théorie est de préciser la manière dont les individus dérivent une conclusion du modèle qu'ils ont construit (Van der Henst, 2000). Les défenseurs de la théorie affirment qu'à partir d'un ensemble de prémisses, les individus essaient d'inférer quelque chose de nouveau ("something new "Johnson-Laird \& Byrne, 1991, page 22), c'est-à-dire une information non triviale. Une question reste 
cependant ouverte : quelle conclusion les individus vont-ils formuler lorsque plusieurs informations non triviales peuvent être inférées d'un même ensemble de prémisses ? On peut tenter de répondre en supposant que les individus vont chercher à produire une information qui engendre le plus d'effets contextuels (en fonction des buts cognitifs de l'individu) pour le plus faible coût de traitement.

La théorie de la pertinence permet également de préciser la procédure de réfutation de la conclusion initiale (Van der Henst, 2000). Les partisans de la théorie des modèles mentaux estiment que le facteur principal qui limite la construction des modèles alternatifs est la faible capacité de la mémoire de travail. Si un modèle alternatif n'est pas construit c'est que la mémoire de travail ne permet pas de le construire. On peut compléter la description de cette étape et faire l'hypothèse que les individus tenteront de construire un modèle alternatif si les effets qu'ils peuvent en escompter satisfont leurs attentes de pertinence. Dès lors, on peut supposer que les individus tenteront de construire un modèle alternatif si l'information qu'il contient peut être considérée comme pertinente ; et qu'ils ne tenteront pas de construire un modèle alternatif non pas seulement dans les situations où la mémoire de travail ne le permet pas mais aussi dans les situations où ils estiment que le modèle alternatif ne peut véhiculer d'information pertinente (Van der Henst, 1999 ; 2000).

\section{Conclusion}

L'un des avantages à développer une approche pragmatique est d'éclairer le débat sur la rationalité. Lorsque l'on adopte une perspective pragmatique dans l'étude du raisonnement et du jugement, on est conduit à réexaminer certains paradigmes expérimentaux et à réviser les conclusions pessimistes sur la qualité des capacités inférentielles auxquelles ont abouti certains chercheurs. Ces derniers ont tendance à développer l'argument suivant: dans la mesure où les tâches à effectuer requièrent des capacités inférentielles, la présence des erreurs ne fait que révéler l'imperfection du système inférentiel et l'incapacité à respecter les normes de validité prévues par la logique classique ou par les principes probabilistes. Mais l'erreur de ces chercheurs est de considérer que la résolution d'une tâche de raisonnement consiste uniquement à appliquer des modèles logiques ou probabilistes aux informations littérales données dans la tâche. Or les tâches de raisonnement sont en très grande majorité des tâches administrées verbalement qui impliquent également une étape interprétative. Evaluer le raisonnement d'un individu nécessite de prendre en compte l'ensemble du processus inférentiel - qui comprend aussi bien la phase de résolution que la phase d'interprétation - à la suite duquel les individus formulent leur réponse. 
L'expérimentateur fonde son évaluation du raisonnement sur l'observation scrupuleuse des principes logiques ou probabilistes mais conçoit ses expériences en négligeant en partie les règles de la communication. Le sujet a finalement de grandes chances d'échouer, car il est en droit de supposer que, dans la plupart des tâches qu'il doit accomplir les informations ont été communiquées par un expérimentateur coopératif. Ce que révèlent les tâches de raisonnement évoquées ici n'est finalement pas tant une limitation des capacités inférentielles qu'une incapacité à s'affranchir des règles pragmatiques. Il faut donc se garder d'établir une déficience inférentielle sur la base d'un échec communicationnel.

En reprenant l'expression de Begg \& Harris (1982), on peut affirmer que les réponses non conformes aux normes logiques des études présentées ici viennent en grande partie du fait que les sujets ont “ joué le jeu de la langue" mais pas “le jeu de la logique”. Or jouer le jeu de la logique n'est pas forcément plus rationnel que jouer le jeu de la langue. Inférer des informations qui sont logiquement dérivables de certaines de mes croyances est même irrationnel si je n'ai pas de bonnes de raisons d'inférer ces informations (Harman, 1995). Supposons que j'entretienne la croyance « $p$ ». De $p$, il s'ensuit logiquement une infinité de conclusions : «p et $\mathrm{p} »$, « $\mathrm{p}$ ou $\mathrm{q} »$, « si non $\mathrm{p}$ alors $\mathrm{q} »$, etc. Si je n'ai pas de raison d'inférer ces conclusions alors il est irrationnel de le faire car ces conclusions vont encombrer inutilement mon esprit : « It is not reasonable or rational to fill your mind with trivial consequences of your beliefs, when you have better things to do with your time, as you often do (Harman, 1995, p. 186)». Par contre, lorsque je suis engagé dans un échange conversationnel et qu'un locuteur me communique quelque chose, je présume qu'il est coopératif. Et il est raisonnable que je lui reconnaisse une intention informative. J'ai donc de bonnes raisons de croire que ce qui est communiqué par ce qu'il dit correspond effectivement à ce qu'il a voulu communiquer, même si le message communiqué n'est pas une conséquence logique de « ce qui est dit». Dans le cas d'une tâche de raisonnement, le sujet serait même irrationnel de penser que l'expérimentateur attend de lui qu'il fasse l'hypothèse que ce qui est communiqué ne correspond pas aux prémisses du raisonnement.

La perspective pragmatique permet d'apprécier toute la dimension communicative des tâches de raisonnement et souligne l'importance de la relation expérimentateur/sujet. A cet égard, elle apporte une description plus fine des processus interprétatifs qui opèrent dans bon nombre de problèmes et peut à l'avenir inciter certains chercheurs à mettre au point des tâches de raisonnement moins " trompeuses ". D'autre part, concevoir des tâches qui tiennent compte des processus liés à la communication peut faciliter l'étude du raisonnement en permettant de circonscrire des processus spécifiquement liés au raisonnement. Cela évite de donner 
naissance à des tâches, initialement conçues pour étudier le raisonnement, mais où seuls opèrent les processus interprétatifs. Enfin, on critique parfois la psychologie du raisonnement (Oléron, 1995) en lui reprochant d'étudier des problèmes trop éloignés de la cognition naturelle. Prendre en compte la dimension pragmatique, c'est reconnaître que les prémisses ne sont pas seulement des entités logiques mais aussi des éléments qui appartiennent au langage humain. Prendre en compte la dimension pragmatique du raisonnement, c'est donc se soucier d'un élément naturel : le langage. Et en ce sens la pragmatique apporte un éclairage écologique (voir aussi Caron, 1983 page 161) sur bon nombres de tâches de raisonnement étudiées jusqu'à maintenant. 


\section{Références bibliographiques}

AHR P.R., YOUNISS, J. - (1970) Reasons for failure on the class inclusion problem. Child Development, $\underline{41}, 132-143$.

ANDERSON J.R., BOWER G.H. - (1973) Human associative memory, New York, Wiley.

BARATGIN J. - (1999) Psychologie du raisonnement probabiliste dynamique: Recherches historiques, pragmatiques et expérimentales sur la règle de Bayes, Thèse de doctorat d'état, Paris, Ecole Polytechnique.

BEGG I., HARRIS G. - (1982) On the interpretation of syllogisms. Journal of Verbal Learning and Verbal Behavior, 21, 595-620.

BONATTI L. - (1994, a) Propositional reasoning by models? Psychological Review, 101, 725 733.

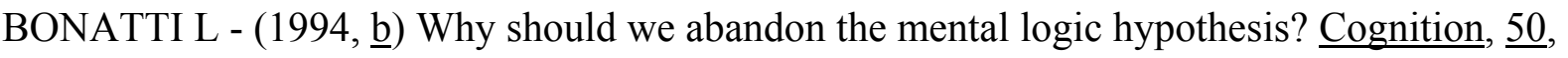
17-39.

BRAINE M.D.S. - (1990) The "natural logic" approach to reasoning, in W.F. OVERTON (Edit.), Reasoning, Necessity and Logic: Developmental perspectives. Hillsdale, (NJ), Lawrence Erlbaum Associates, 133-157.

BRAINE M.D.S., O'BRIEN D.P. - (1991) A theory of If: A lexical entry, reasoning program and pragmatic principles. Psychological Review, $\underline{98}, 182-203$.

BRAINE M.D.S., O'BRIEN D.P. (Edit.) - (1998) Mental Logic, Mahwah, (NJ), Lawrence Erlbaum Associates.

BRAINE M.D.S., REISER B.J, RUMAIN B. - (1984) Some empirical justification for a theory of natural propositional logic, in G.H. BOWER (Edit.), The psychology of learning and motivation, Vol. 18, New York, Academic Press, 315-371.

BRAINE, M.D.S., RUMAIN, B. (1983) Logical Reasoning. Dans J.H. Flavell, \& E.M. Markman (Edit). Carmichael's handbook of child psychology, Vol. 3: Cognitive development. New York: John Wiley.

BYRNE R.M.J. - (1989) Suppressing valid inferences with conditionals. $\underline{\text { Cognition, }} \underline{\text { 31, }}$ 61-83.

CARON J. - (1983) Les Régulations du discours, psycholinguistique et pragmatique du langage, Paris, PUF.

CHAN D., CHUA F. - (1994) Suppression of valid inferences: syntactic views, mental models, and relative salience. Cognition, $\underline{53}, 217-238$. 
CHENG P.W., HOLYOAK K.J. - (1985) Pragmatic reasoning schemas. Cognitive Psychology, 17, 391-416.

CLARK H.H. - (1969) Linguistic processes in deductive reasoning. Psychological Review, $\underline{76}$, $387-404$.

CLARK H.H., MARSHALL C.E. - (1981) Definite reference and mutual knowledge, in A.K. JOSHI, B.L. WEBBER et I.A. SAG. (Edit.), Linguistics structure and discourse setting, Cambridge, Cambridge University Press, 10-63.

CLARK H.H., MURPHY G.L. - (1982) La visée vers l'auditoire dans la signification et la référence. Bulletin de Psychologie, 35, 767-776.

COHEN L.J. - (1981) Can human irrationality be experimentally demonstrated? The Behavioral and Brain Sciences, 4 , 317-370.

COLE M., SCRIBNER S. - (1974) Culture thought: A psychological introduction. New York, Wiley.

DE SOTO C.B., LONDON M., HANDEL S. - (1965) Social reasoning and spatial paralogic. Journal of Personality and Social Psychology, 2, 293-307.

DONALDSON M. - (1982) Conservation: What is the question? British Journal of Psychology, 73, $199-207$.

DUCROT O. - (1972) Dire et ne pas dire, Paris, Hermann.

DULANY D.L., HILTON D.J. - (1991) Conversational implicature, conscious representation and the conjunction fallacy. Social Cognition, $\underline{9}, 85-100$.

EVANS J.ST.B.T. - (1982) The psychology of deductive reasoning, London, Routledge and Keagan Paul.

EVANS J.ST.B.T. - (1989) Bias in human reasoning: Causes and consequences, Hove, UK, Lawrence Erlbaum Associates.

EVANS J.ST.B.T. - (1991) Theories of Human reasoning: The Fragmented state of the Art. Theory and Psychology, 1, 83105.

EVANS J.ST.B.T., NEWSTEAD, S.E., BYRNE, R.M.J. - (1993) Human reasoning. The Psychology of Deduction, Hove, UK, Lawrence Erlbaum Associates.

EVANS J. ST.B.T., OVER, D. - (1996) Rationality and Reasoning, Lawrence Erlbaum Associates.

FILLENBAUM S. - (1971) On coping with ordered and unordered conjunctive sentences. Journal of Experimental Psychology, 87, 93-98.

FILLENBAUM S. - (1974, a) Pragmatic normalization: further results for some conjunctive and disjunctive sentences. Journal of Experimental Psychology, 102, 574-578. 
FILLENBAUM S. - (1974, ㅁ) Or: Some uses. Journal of Experimental Psychology, 103, 913 921.

FILLENBAUM S. - (1977) Mind your p's and q's: the role of content and context in some uses of and, or, and if, in G.H. BOWER (Edit.), The psychology of learning and motivation, Vol. 11, New York, Academic Press, 41-100.

FILLENBAUM S. - (1978) How to do some things with IF, in J.W. COTTON et R.L. KLATZKY (Edit.), Semantic factors in cognition, Hillsdale, (NJ), Lawrence Erlbaum Associates, 168-212.

FILLENBAUM S. - (1993) Deductive reasoning: What are taken to be the premises and how are they interpreted? The Behavioral and Brain Sciences, 16, 348-349.

GEIS M.C., ZWICKY A.M. - (1971) On invited inference. Linguistic Inquiry, 2, 561-566.

GEORGE C. - (1997) Polymorphisme du raisonnement humain: Une approche de la flexibilité de l'activité inférentielle, Paris, PUF.

GIROTTO V. - (1995) Sur les fondements de la théorie des modèles mentaux: à propos de l'article de P. Oléron. L’Année Psychologique, 95, 707-715.

GIROTTO V., KEMMELMEIER, M., SPERBER, D., VAN DER HENST, J.B. - (2001) Inept reasoners or pragmatic virtuosos? Relevance and the deontic selection task. Cognition, $\underline{81}$, B69-B76.

GRICE H.P. - (1975) Logic and conversation, in P. COLE et J.L. MORGAN (Edit.), Studies in syntax, Vol. 3, Speech acts, New York, Academic Press.

GRIGGS R.A., COX J.R. - (1982) The elusive thematic-materials effect in Wason's selection task. British Journal of Psychology, 35, 519-533.

GRIGGS R.A., NEWSTEAD S.E. - (1983) Drawing inferences from quantified statements: A study of the square of opposition. Journal of Verbal Learning and Verbal Behavior, 22, $535-546$

HAMILL J. - (1990) Ethno-logic: The anthropology of human reasoning, Urbana, University of Illinois Press.

HARDMAN D. - (1998) Does reasoning occur on the selection task? A comparison of relevance-based theories. Thinking and reasoning, 4, 353-376.

HARMAN G. - (1995) Rationality, in E.E. SMITH et D. N. OSHERSON (Edit.), Thinking: An invitation to cognitive science. $2^{\text {ième }}$ ed., MIT Press.

HENLE M. - (1962) The relation between logic and thinking. Psychological Review, 69, 366378. 
HENLE M. - (1978) Foreword to R. Revlin and R.E. Mayer (Edit.), Human Reasoning. Washington, (DC), Winston.

HILTON D.J. - (1995) The social context of reasoning: Conversational inference and rational judgment. Psychological Bulletin, 118, 248-271.

HORN L.R. - (1972) On the semantic properties of the Logical Operators in English. Mimeo. Indiana University Linguistics Club.

HUTTENLOCHER J. - (1968) Constructing spatial images: a strategy in reasoning. Psychological Review, 75, 550-560.

JOHNSON-LAIRD P.N. - (1983) Mental Models. Cambridge, Cambridge University Press.

JOHNSON-LAIRD P.N., BYRNE R.J.M. - (1991) Deduction, Hove, U.K, Lawrence Erlbaum Associates.

JOHNSON-LAIRD P.N., BYRNE R.M.J., SCHAEKEN W. - (1992) Propositional reasoning by model. Psychological Review, 99, 418-439.

JOHNSON-LAIRD P.N., BYRNE R.M.J., SCHAEKEN W. - (1994) Why models rather than rules give a better account of propositional reasoning: a reply to Bonatti and to O'Brien, Braine and Yang. 101, 734-739.

KAHNEMAN D., SLOVIC P., TVERSKY A. - (1982) Judgment under Uncertainty: Heuristics and Biases, New York, Cambridge University Press.

KAHNEMAN D., TVERSKY A. - (1973) On the psychology of prediction. Psychological $\underline{\text { Review, }}$ 80, 237-251.

KLAYMAN J., HA Y.W. - (1987) Confirmation, disconfirmation and information in hypothesis testing. Psychological Review, 94, 211-228.

KOEHLER J.J. - (1996) The base rate fallacy reconsidered: descriptive, normative, and methodological challenges. Behavioral and Brain Sciences, 19, 1-53.

KRAUSS R. M., FUSSELL S. R. - (1996) Social psychological approaches to the study of communication, in E. T. HIGGINS et A. KRUGLANSKI (Edit.), Social psychology: Handbook of basic principles. New York, Guilford Press.

KROSNICK J.A., LI, F., LEHMAN D.R., - (1990) Conversational conventions, order of information acquisition, and the effect of base rates and individuating information on social judgments. Journal of Personality and Social Psychology, 59, 1140-1152.

LEVINSON S.C. - (1983) Pragmatics, New York, Cambridge University Press.

LURIA A.R. - (1976) Cognitive development: Its cultural and social foundations, Cambridge, (MA), Harvard University Press. 
MCGARRIGLE J., DONALDSON M. - (1975) Conversational accidents. $\underline{\text { Cognition, }} \underline{3}, 341$ 350.

MACCHI L. - (1995) Pragmatic aspects of the base-rate fallacy. Quarterly Journal of Experimental Psychology, $\underline{48}, 188-207$.

MANKTELOW K.I., OVER D.E. (Edit.),- (1993) Rationality: Psychological and Philosophical Perspectives, London, Routledge.

MARKMAN E. - (1973) The facilitation of part-whole comparisons by use of the collective noun "family." Child-Development, $\underline{44}, 837-840$

NEWSTEAD S.E. - (1989) Interpretational errors in syllogistic reasoning. Journal of Memory and Language, $\underline{28}, 78-91$.

NOVECK I.A. - (2001) When children are more logical than adults: Investigations of scalar implicature. Cognition, $\underline{78}, 165-188$.

NOVECK I.A., LEA R.B., DAVIDSON G.M., O’BRIEN, D.P. - (1991) Human reasoning is both logical and pragmatic. Intellectica, 11, 81-109.

NOVECK I., POLITZER, G. - (1998) Leveling the playing field, in M.D.S. BRAINE et D.P. O’BRIEN, (Edit.), Mental Logic, Mahwah, (NJ) Lawrence Erlbaum Associates. 367-384.

O'BRIEN D.P., BRAINE M.D.S., YANG Y. - (1994) Propositional reasoning by models?

Simple to refute in principle and in practice. Psychological Review, 101, 711-724.

O’BRIEN D.P. DAVIDSON G.M. - (1989) Evaluation of evidence for sufficiency, for necessity and for necessity-and-sufficiency. Quarterly Journal of Experimental Psychology, $\underline{41 \mathrm{~A}}, 531-551$.

OLÉRON P. - (1995) Un examen critique de la théorie des modèles mentaux. L’Année Psychologique, 95, 693-706.

PIAGET J. INHELDER B. - (1959) La genèse des structures logiques élémentaires, Neuchâtel, Delachaux \& Niestlé.

POLITZER G. - (1986) Laws of language use and formal logic. Journal of Psycholinguistic $\underline{\text { Research, }}$ 15, 47-92.

POLITZER G. - (1990) Immediate deduction between quantified sentences, in K.J. GILHOOLY, M.T.G. KEANE, R.H. LOGIE et G. ERDOS (Edit.), Lines of thinking. Reflections on the psychology of thought, Vol. 1, Chichester, UK, Wiley, 85-97. POLITZER G. - (1991) L'informativité des énoncés: contraintes sur jugement et le raisonnement. Intellectica, $\underline{11}, 111-147$.

POLITZER G. - (1993) La psychologie du raisonnement: Lois de la pragmatique et logique formelle, Thèse de doctorat d'état ès lettres et sciences humaines. 
POLITZER G., BRAINE M.D.S. - (1991) Responses to inconsistent premises cannot count as suppression of valid inferences. Cognition, $\underline{38}, 103-108$.

POLITZER G., NOVECK I.A. - (1991) Are conjunction rule violations the result of conversational rule violations. Journal of Psycholinguistic Research, 20, 83-103.

RIPS L.J. - (1983) Cognitive processes in propositional reasoning. Psychological Review, 90, $38-71$.

RIPS L.J. - (1994) The psychology of proof, London, M.I.T Press.

ROSSI, S., CAVERNI, J.P., GIROTTO, V. (2001) Hypothesis testing in a rule discovery problem: When a focused procedure is effective.

RUMAIN, B., CONNELL, J., \& BRAINE, M.D.S. (1983) Conversational comprehension processes are responsible for reasoning fallacies in children and adults. Developmental Psychology, 19, 471-481.

SCHWARTZ N., STRACK F., HILTON D. NADERER G. - (1991) Base rates, representativeness, and the logic of conversation: The contextual relevance of "irrelevant" information. Social Cognition, $\underline{9}$, 67-84.

SMITH L.B. RIZZO T.A. - (1982) Children's understanding of the referential properties of

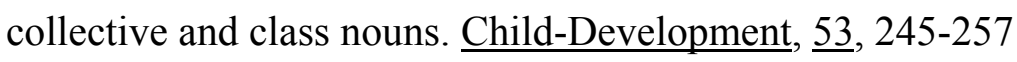

SPERBER D., WILSON D. - (1986/1995) Relevance: Communication and Cognition, Oxford, Blackwell. Traduction française A. Gerschenfeld, D. Sperber, La pertinence: Communication et cognition, Paris, Minuit. - (1989)

SPERBER D., CARA F., GIROTTO V. - (1995) Relevance theory explains the selection task. Cognition, 57, 31-95.

SPINOZA B. - (1677/1974) L'Ethique, Paris, La Pléiade.

STICH S.P. - (1990) The fragmentation of reason, Cambridge, MIT Press.

TVERSKY A., KAHNEMAN D. - (1980) Causal schemata in judgments under uncertainty, in M. FISHBEIN (Edit.), Progress in Social Psychology, Vol. 1, Hillsdale, (NJ) Erlbaum, 4972 .

TVERSKY A., KAHNEMAN D. - (1983) Extensional versus intuitive reasoning: The conjunction fallacy in probability judgment. Psychological Review, 90, 293-315.

TWENEY R.D., DOHERTY M.E., WORNER W.J., PLISKE D.B., MYNATT C.R. GROSS et K.A., ARKKELIN D.L. - (1980) Strategies of rule discovery on an inference task. Quarterly Journal of Experimental Psychology, 32, 109-123. 
VAN DER HENST J.B. - (1999) The mental model theory and spatial reasoning re-examined: the role of relevance in premise order. British Journal of Psychology, 90, 73-84.

VAN DER HENST J.B. - (2000) Mental model theory and pragmatics. Behavioral and Brain Sciences, $\underline{23}, 283-284$.

WASON, P.C. - (1960) On the failure to eliminate hypotheses in a conceptual task. Quarterly Journal of Experimental Psychology, 12, 129-140.

WASON, P.C. - (1966) Reasoning, in B.M. FOSS (Edit.), New Horizons in Psychology, Vol 1. Harmondsworth, Penguin.

WASON, P.C. - (1968) Reasoning about a rule. Quarterly Journal of Experimental Psychology, $\underline{20}, 273-281$. 


\section{Notes}

Note 1.

Notons cependant qu'il ne sera pas fait ici mention des tests d'intelligence qui utilisent parfois des problèmes de raisonnement non verbal.

Note 2.

On peut noter que les remarques de Henle sur la difficulté des sujets à considérer la tâche de raisonnement comme une tâche purement formelle rejoignent les analyses faites par les chercheurs qui travaillent dans le domaine de la psychologie interculturelle (Cole Scribner, 1974, chapitre 7 ; Luria, 1976, chapitre 4 ; Hamill, 1990 ) Ces chercheurs remarquent que les individus appartenant aux sociétés traditionnelles ont tendance à transformer le problème posé de sorte que les prémisses deviennent compatibles avec leur expérience quotidienne du monde. 